\title{
Etalonnages du taux de croissance du PIB français sur la base des enquêtes de conjoncture
}

\author{
Marie BESSEC
}

\begin{abstract}
Résumé
Cet article développe des étalonnages du taux de croissance du PIB français destinés à produire des prévisions de très court terme de l'activité. Ils sont construits exclusivement à partir de données d'enquête de l'Insee, dans l'industrie mais également dans les services et le bâtiment. Nous examinons deux stratégies de réduction de l'information, l'une fondée sur l'algorithme de sélection automatique GETS par blocs de Hendry et Krolzig (2005), l'autre sur la méthode de combinaison popularisée par Stock et Watson (2004). Ces deux méthodes sont évaluées hors échantillon au travers de régressions récursives et roulantes. Nous montrons la supériorité des étalonnages construits avec GETS et l'intérêt de considérer d'autres enquêtes que celle dans l'industrie dans les stratégies de modélisation et de prévision.
\end{abstract}

Mots-Clefs : Prévision du PIB, étalonnages.

Classification JEL : C22, E32, E37.

\section{Introduction}

Cet article a pour objectif de construire des étalonnages du taux de croissance du Produit Intérieur Brut français afin de prévoir les fluctuations de très court terme de l'activité.

La prévision du taux de croissance trimestriel du PIB comporte plusieurs difficultés. L'ensemble d'information évolue tout d'abord au cours du trimestre, car les variables explicatives utilisées sont pour la plupart mensuelles et réactualisées tous les mois. Elles sont par ailleurs très nombreuses et un modèle ne pouvant contenir qu'un nombre limité de variables explicatives, se pose le problème de la synthèse de l'information apportée par ces variables. Il existe trois approches principales pour synthétiser l'information: l'analyse factorielle statique ou dynamique ${ }^{1}$, la méthode de combinaison $^{2}$ ou des algorithmes de sélection automatique des variables explicatives. Nous considérons ici la méthode de combinaison et l'algorithme de sélection automatique GETS $^{3}$. Ces deux méthodes sont

\footnotetext{
* LEDA-Université Paris Dauphine, étude réalisée pour la Direction Générale du Trésor et de la Politique Economique. Je tiens à remercier X. Bonnet, O. Bouabdallah, S. Dali, W. Roos, C. Simon, ainsi que deux rapporteurs anonymes pour leurs commentaires et suggestions. Toutes les erreurs et omissions pouvant subsister relèvent de mon entière responsabilité.

${ }^{1}$ Voir notamment Stock et Watson (2002) en statique, Forni et al. (2000, 2005) en dynamique.

${ }^{2}$ Cette méthode est historiquement due aux travaux de Reid (1968) et de Bates et Granger (1969) et a été popularisée par Stock et Watson $(1999,2004)$ notamment.

${ }^{3}$ Cet outil a été proposé par Hoover et Perez (1999) et étendu par Hendry et Krolzig (1999, 2001). Nous allons considérer ici une variante de cette méthode décrite par Hendry et Krolzig (2005).
} 
adaptées suivant Dubois et Michaux (2006) pour prendre en compte l'évolution de l'ensemble d'information au cours du trimestre.

Nous avons choisi de restreindre l'ensemble d'information aux soldes d'opinion issus des enquêtes de conjoncture de l'Insee. Au travers de questions posées aux chefs d'entreprise sur l'activité passée et à venir, elles donnent des indications sur les évolutions de court terme de l'activité. Nous excluons en revanche les variables financières et macro-économiques parmi lesquelles les composantes du PIB connues au cours du trimestre (l'indice de production industrielle, la consommation manufacturière, etc) et les variables affectant plus indirectement le PIB (le taux de change, le prix du pétrole, les taux d'intérêt, etc). L'idée est ici d'identifier le signal émis par les enquêtes de conjoncture sur l'activité et d'en évaluer la fiabilité. Les données d'enquête ont en outre l'avantage d'être disponibles rapidement et d'être peu révisées. Les prévisions qui seront obtenues à partir des outils développés dans cette étude auront ainsi l'avantage d'être définitives. Elles couvrent par ailleurs la plupart des secteurs d'activité. Nous considérons ici les enquêtes relatives à l'industrie, mais également aux services et au bâtiment.

L'approche retenue se rapproche de plusieurs travaux existants. Notre étude s'inscrit tout d'abord dans un courant récent de la littérature qui remet en cause l'utilisation exclusive des indicateurs portant sur le seul secteur industriel dans l'analyse conjoncturelle et qui avance que les enquêtes de conjoncture réalisées dans les autres secteurs d'activité, dans les services notamment, peuvent avoir un apport dans la prévision de l'activité française (Bouton et ErkelRousse, 2002, Erkel-Rousse et Prioux, 2002, Darné et Brunhes-Lesage, 2007 et Erkel-Rousse et Minodier, 2008). Nous innovons toutefois en utilisant l'enquête dans le bâtiment. Ce secteur d'activité est rarement pris en compte $^{4}$ ce qui peut surprendre compte tenu du caractère relativement cyclique de l'immobilier.

Nous innovons également dans le choix de la technique de réduction de l'information. Si l'algorithme de sélection automatique GETS est de plus en plus utilisé (il est par exemple appliqué directement aux soldes d'opinion par Erkel-Rousse et Minodier en 2008 ou aux facteurs tirés d'analyses en composantes principales par Darné et Brunhes-Lesage en 2007), c'est moins le cas de la méthode de combinaison qui n'a pas encore fait l'objet d'étude systématique pour la prévision du taux de croissance du PIB français sur de courts horizons ${ }^{5}$. Notre étude se démarque également de la littérature existante par l'utilisation de la variante par blocs de la méthode GETS (Hendry et Krolzig, 2005). Cette approche permet d'envisager un plus grand nombre de variables explicatives potentielles en appliquant l'algorithme de sélection à des blocs de variables explicatives constitués de façon aléatoire.

Les performances de ces méthodes en matière de prévision sont évaluées hors échantillon au travers de régressions récursives et roulantes. Nous mesurons la qualité des prédicteurs sur la

\footnotetext{
${ }^{4}$ L'OFCE et la Banque de France font exception à ce constat. L'OFCE fonde ses prévisions de l'activité sur une équation comportant comme variables explicatives des données "dures" - le taux de change réel du dollar contre l'euro, la pente des taux et le prix réel du pétrole - et des données d'enquêtes - les premiers facteurs de trois ACP, l'une sur l'enquête réalisée par l'Insee dans l'industrie, la deuxième dans le bâtiment et la dernière dans les services (Heyer et Péléraux, 2004). Depuis la fin de l'année 2008, l'OFCE intègre également dans cette équation la confiance des ménages dans la situation économique mesurée par l'enquête Insee auprès des ménages (Heyer et al., 2008). L'approche développée par la Banque de France dans son modèle OPTIM de prévision à courtterme de l'activité française (voir Bahroumi et al., 2008, pour une présentation de la version révisée de la maquette) intègre également le secteur de la construction de manière explicite.

${ }^{5}$ On trouve en revanche des applications récentes de cette méthode au PIB de la zone euro (Angelini et al., 2008, Drechsel et Maurin, 2008) ou américain (Kitchen et Monaco, 2003).
} 
base des critères RMSFE et MAFE usuels, ainsi que sur l'aptitude des modèles à anticiper les phases d'accélération ou de décélération du PIB et la localisation du taux de croissance par rapport au rythme de croissance tendanciel. L'évaluation est réalisée sur la série actuelle de PIB et sur les premières publications qui peuvent différer fortement. Comme les études antérieures, nous trouvons un apport significatif des données d'enquête pour estimer et prévoir le taux de croissance sur les horizons de prévision courts relativement à un processus autorégressif simple. Les soldes d'opinion de l'enquête dans l'industrie comme dans les services et le bâtiment interviennent dans les étalonnages les plus performants. Nos résultats sont par ailleurs en faveur de l'utilisation de la méthode de sélection automatique, plus performante sur les horizons de prévision courts même si le gain est plus évident sur les comptes définitifs que sur les premières publications du taux de croissance du PIB.

Cette étude est organisée comme suit. La première section décrit les données et les deux techniques de prévision concurrentes. La deuxième section présente les variables sélectionnées et la qualité d'ajustement dans les deux méthodes. La troisième section expose les résultats d'évaluation hors échantillon. La dernière section conclut.

\section{Données et méthodes}

\subsection{Les données}

La variable que l'on cherche à prévoir est le taux de croissance trimestriel du PIB réel aux prix de l'année précédente chaînés mesuré par l'Insee. Les variables explicatives utilisées proviennent des enquêtes de conjoncture réalisées par l'Insee (il existe aujourd'hui une quinzaine d'enquêtes de conjoncture qui couvrent la plupart des secteurs d'activité, cf. annexe 1 pour une revue des enquêtes infra-annuelles). Les données utilisées sont corrigées des variations saisonnières.

Les données d'enquête sont très utilisées en analyse conjoncturelle. Elles ont l'avantage d'être disponibles très rapidement (dès la fin du mois concerné) contrairement aux indicateurs quantitatifs (l'indice de production industrielle est par exemple publié 40 jours après la fin du mois concerné et la consommation manufacturière 22 jours après) et d'être peu révisées (une seule fois lors de la publication suivante pour tenir compte notamment des réponses reçues tardivement) ${ }^{6}$. Nous avons choisi d'exclure les autres sources d'information (indicateurs macroéconomiques et financiers). L'idée est ici d'identifier le signal émis par les enquêtes de conjoncture sur l'activité et d'en évaluer la fiabilité. On a également ainsi la possibilité d'obtenir des prévisions "définitives" non sensibles aux révisions des indicateurs quantitatifs habituellement utilisés dans les étalonnages.

La période d'étude s'étend du deuxième trimestre de 1988 au quatrième trimestre de 2006 et comprend 75 observations. La période ne débute qu'en 1988 en raison de l'utilisation de l'enquête dans les services initiée au premier trimestre de l'année 1988. Nous avons par ailleurs exclu les dernières observations de l'analyse (2007 à 2008) car le taux de croissance du PIB y est susceptible d'être révisé (en toute rigueur, il aurait également fallu écarter les observations de l'année 2006) ${ }^{7}$. Nous avons utilisé, pour le PIB comme pour les variables d'enquêtes, les séries disponibles au moment de la rédaction de cette étude à la fin du mois de

\footnotetext{
${ }^{6}$ La désaisonnalisation de la série est également refaite une fois par an.

${ }^{7}$ Les comptes trimestriels ne sont définitifs que trois ans après leur première publication et donnent lieu auparavant à de fréquentes révisions.
} 
février 2009 et non les données qui auraient été disponibles en temps réel, c'est-à-dire au moment de la prévision. Comme on l'a mentionné plus haut, les révisions des enquêtes de conjoncture sont de faible ampleur et doivent avoir peu d'incidence, celles du PIB plus importantes comme on l'a vu récemment ${ }^{8}$, jouent certainement davantage ${ }^{9}$.

En pratique toutefois, les modèles ne sont estimés qu'à partir des comptes définitifs, i.e. en excluant les observations des trois dernières années. Les révisions de PIB doivent alors peu affecter les prévisions obtenues dès lors que les modèles n'intègrent pas de termes autorégressifs. En contrepartie, on prévoit vraisemblablement plutôt le chiffre définitif de croissance de PIB que les premiers résultats. Afin d'examiner ce dernier point, nous évaluerons également nos prévisions à la lumière des premiers résultats (i.e. des taux de croissance trimestriels du PIB publiés par l'Insee deux mois après le trimestre).

Tableau 1. Les soldes d'opinion utilisés dans l'analyse

\begin{tabular}{|c|c|c|c|c|c|}
\hline & Solde & Fréq. & Transform. & Notation & Parution \\
\hline \multirow{7}{*}{ 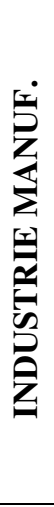 } & Production passée & M & Niveau, $\Delta$ & $P P A_{m j}^{m a n}, P P A_{m j}^{m a n}-P P A_{m j-1}^{m a n}$ & $\mathrm{M}+0$ \\
\hline & Perspectives personnelles de prod. & M & Niveau, $\Delta$ & $P P E R_{m j}^{m a n}, P P E R_{m j}^{m a n}-P P E R_{m j-1}^{m a n}$ & $\mathrm{M}+0$ \\
\hline & Perspectives générales de prod. & M & Niveau, $\Delta$ & $P G E N_{m j}^{m a n}, P G E N_{m j}^{m a n}-P G E N_{m j-1}^{m a n}$ & $\mathrm{M}+0$ \\
\hline & Carnets de commandes globaux & M & Niveau, $\Delta$ & $C C_{m j}^{m a n}, C C_{m j}^{m a n}-C C_{m j-1}^{m a n}$ & $\mathrm{M}+0$ \\
\hline & Carnets de commandes étrangers & M & Niveau, $\Delta$ & $C C E_{m j}^{m a n}, C C E_{m j}^{m a n}-C C E_{m j-1}^{m a n}$ & $\mathrm{M}+0$ \\
\hline & Niveau des stocks & M & Niveau, $\Delta$ & $S T O_{m j}^{m a n}, S T O_{m j}^{m a n}-S T O_{m j-1}^{m a n}$ & $\mathrm{M}+0$ \\
\hline & Demande prévue & Q & Niveau, $\Delta^{T}$ & $D A_{Q}^{m a n}, D A_{Q}^{m a n}-D A_{Q-1}^{m a n}$ & $\begin{array}{l}\mathrm{Q}+0 \\
(\mathrm{M} 1)\end{array}$ \\
\hline \multirow{5}{*}{ 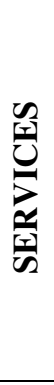 } & Activité passée & M & Niveau, $\Delta$ & $C A P A_{m j}^{s v}, C A P A_{m j}^{s v}-C A P A_{m j-1}^{s v}$ & $\mathrm{M}+0$ \\
\hline & Activité prévue & M & Niveau, $\Delta$ & $C A P R E_{m j}^{s v}, C A P R E_{m j}^{s v}-C A P R E_{m j-1}^{s v}$ & $\mathrm{M}+0$ \\
\hline & Résultat d'exploitation passé & Q & Niveau, $\Delta^{T}$ & $R E P A_{Q}^{S V}, R E P A_{Q}^{s v}-R E P A_{Q-1}^{S V}$ & $\begin{array}{l}\mathrm{Q}+0 \\
(\mathrm{M} 1) \\
\end{array}$ \\
\hline & Résultat d'exploitation prévu & Q & Niveau, $\Delta^{T}$ & $R E P R E_{Q}^{s v}, R E P R E_{Q}^{S V}-R E P R E_{Q-1}^{S V}$ & $\begin{array}{l}\mathrm{Q}+0 \\
(\mathrm{M} 1)\end{array}$ \\
\hline & Demande prévue & Q & Niveau, $\Delta^{T}$ & $D A_{Q}^{s v}, D A_{Q}^{s v}-D A_{Q-1}^{s v}$ & $\begin{array}{l}\mathrm{Q}+0 \\
(\mathrm{M} 1)\end{array}$ \\
\hline \multirow{6}{*}{ 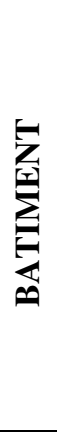 } & Activité passée & M & Niveau, $\Delta$ & $P P A_{m j}^{b a t}, P P A_{m j}^{b a t}-P P A_{m j-1}^{b a t}$ & $\mathrm{M}+0$ \\
\hline & Activité prévue & M & Niveau, $\Delta$ & $P P E R_{m j}^{b a t}, P P E R_{m j}^{b a t}-P P E R_{m j-1}^{b a t}$ & $\mathrm{M}+0$ \\
\hline & Carnets de commandes & M & Niveau, $\Delta$ & $C C_{m j}^{b a t}, C C_{m j}^{b a t}-C C_{m j-1}^{b a t}$ & $\mathrm{M}+0$ \\
\hline & Effectifs passés & M & Niveau, $\Delta$ & $E F P A_{m j}^{b a t}, E F P A_{m j}^{b a t}-E F P A_{m j-1}^{b a t}$ & $\mathrm{M}+0$ \\
\hline & Effectifs prévus & M & Niveau, $\Delta$ & $E F P R E_{m j}^{b a t}, E F P R E_{m j}^{b a t}-E F P R E_{m j-1}^{b a t}$ & $\mathrm{M}+0$ \\
\hline & $\begin{array}{l}\text { Taux d'utilisation des capacités de } \\
\text { production }\end{array}$ & M & Niveau, $\Delta$ & $T U C_{m j}^{b a t}, T U C_{m j}^{b a t}-T U C_{m j-1}^{b a t}$ & $\mathrm{M}+0$ \\
\hline
\end{tabular}

Lecture : L'extension mj, $j=1,2$ ou 3, fait référence au mois $j$ du trimestre en cours et $Q$ au trimestre en cours. $\Delta$ désigne une variation mensuelle et $\Delta^{T}$ une variation trimestrielle. Dans la dernière colonne, la notation $M+O(Q+0)$ indique que la variable paraît au cours du mois (du trimestre) concerné.

\footnotetext{
${ }^{8}$ L'estimation de la croissance annuelle du PIB en volume en 2005 a par exemple été revue successivement de $1,2 \%$ à $1,7 \%$ puis à $1,9 \%$. Le taux de croissance trimestriel du PIB au premier trimestre 2008 a quant à lui été révisé de $0,64 \%$ à $0,40 \%$ entre mai 2008 et février 2009.

${ }^{9}$ On pourra se référer à Croushore (2006) pour une revue récente de l'impact de l'utilisation des données en temps réel sur l'évaluation des prévisions.
} 
Les soldes d'opinion retenus dans l'analyse sont présentés dans le tableau 1. Ils se réfèrent à trois secteurs d'activité distincts : l'industrie manufacturière, le bâtiment et les services ${ }^{10}$. Nous avons en revanche écarté les enquêtes sur le commerce de détail et le commerce de gros. Nous avons considéré les principaux soldes des trois enquêtes retenus dans la construction des indicateurs synthétiques des climats des affaires sectoriels ${ }^{11}$. Les soldes considérés sont de fréquence mensuelle ${ }^{12}$, à l'exception des soldes relatifs à la demande attendue dans l'industrie et les services et des résultats d'exploitation passé et prévu dans les services de fréquence trimestrielle. Les soldes sont considérés en niveau et en variation mensuelle ${ }^{13}$. Le PIB étant une variable trimestrielle, les séries mensuelles sont "découpées" en trois séries trimestrielles, la première étant constituée des valeurs prises par le solde d'opinion aux mois $1 \mathrm{du}$ trimestre, la deuxième aux mois 2 et la troisième aux mois 3 , comme on le verra ci-après.

Nous intégrerons également des termes autorégressifs dans les équations si leur inclusion améliore la spécification (i.e. s'ils sont sélectionnés par l'algorithme de sélection automatique ou s'ils conduisent à une amélioration des critères d'information dans la méthode de combinaison, comme nous le verrons dans la suite).

\subsection{La prise en compte de l'évolution de l'information dans le trimestre}

L'ensemble d'information à la disposition du conjoncturiste pour construire ses prévisions évolue au cours du trimestre. Ainsi, au mois 1, on ne dispose que des soldes d'opinion au mois $1 \mathrm{du}$ trimestre en cours et on ne pourra utiliser que les enquêtes des mois 2 et 3 des trimestres précédents. En revanche, au mois 3, on pourra utiliser les enquêtes parues tous les mois du trimestre en cours (et des trimestres précédents). Il est pertinent de prendre en compte cette évolution, car les agents voient de plus en plus clair sur la production à mesure que l'on avance dans le trimestre; aussi les dernières enquêtes fournissent-elles des signaux de meilleure qualité pour prévoir l'activité (comme on le verra à travers la qualité d'ajustement et prédictive des modèles).

Pour prendre en compte l'évolution de l'ensemble d'information, nous suivons l'approche de Dubois et Michaux (2006) consistant à utiliser des étalonnages différents suivant le mois auquel on se situe au moment de la prévision. Chacun d'entre eux est construit sur la base des seuls soldes connus au mois concerné. Nous exploitons l'information disponible sur les trois derniers mois connus au moment de la prévision : les soldes en niveau au mois en cours et les variations des soldes sur les trois derniers mois. Plus formellement, en notant $X^{i}, i=1, \ldots, 14$ les 14 soldes mensuels présentés dans le tableau 1 et $Y_{Q}^{j}, j=1, \ldots, 4$, les 4 soldes trimestriels, nos prédicteurs sont construits sur la base des ensembles d'information suivants :

\footnotetext{
${ }^{10}$ On utilise les soldes portant sur l'ensemble des services, formé des services aux entreprises et des services aux particuliers.

${ }^{11}$ Les effectifs prévus du bâtiment utilisés ici n'interviennent pas dans le calcul de l'indicateur synthétique du bâtiment, tandis que les perspectives générales dans les services non prises en compte car disponibles sur une période trop courte interviennent en revanche dans le calcul de l'indicateur synthétique dans les services.

${ }^{12}$ Dans le cas des enquêtes portant sur le bâtiment, les enquêtes sont trimestrielles jusqu'au troisième trimestre de 1993 (et les soldes publiés au premier mois du trimestre). De même, l'enquête dans les services est trimestrielle du premier trimestre de 1988 (et non produite auparavant) au deuxième trimestre de 2000. Nous avons recouru pour ces variables à des techniques d'interpolation - un spline cubique - afin d'obtenir des séries mensuelles sur l'ensemble de la période considérée.

${ }^{13}$ On utilisera la variation trimestrielle des quatre soldes de fréquence trimestrielle : la demande prévue dans l'industrie et les services et les résultats d'exploitation passé et prévu dans les services.
} 
Au mois $1: X_{m 1}^{i}, X_{m 1}^{i}-X_{m 3(-1)}^{i}, X_{m 3(-1)}^{i}-X_{m 2(-1)}^{i}, X_{m 2(-1)}^{i}-X_{m 1(-1)}^{i}, Y_{Q}^{j}, Y_{Q}^{j}-Y_{Q-1}^{j}$

Au mois $2: X_{m 2}^{i}, X_{m 2}^{i}-X_{m 1}^{i}, X_{m 1}^{i}-X_{m 3(-1)}^{i}, X_{m 3(-1)}^{i}-X_{m 2(-1)}^{i}, Y_{Q}^{j}, Y_{Q}^{j}-Y_{Q-1}^{j}$

Au mois $3: X_{m 3}^{i}, X_{m 3}^{i}-X_{m 2}^{i}, X_{m 2}^{i}-X_{m 1}^{i}, X_{m 1}^{i}-X_{m 3(-1)}^{i}, Y_{Q}^{j}, Y_{Q}^{j}-Y_{Q-1}^{j}$

avec $i=1, \ldots, 14$ et $j=1, \ldots, 4$.

La variable endogène n'est connue que 45 jours après la fin du trimestre concerné, soit au milieu du deuxième mois du trimestre suivant, de sorte qu'il est utile d'avoir un modèle d'estimation au mois 1 du taux de croissance du PIB du trimestre précédent. Dans le cas de cette prévision, les soldes prospectifs du mois 1 et leur variation sont retardés (i.e. on considère dans la régression les soldes et la variation des soldes au mois $1 \mathrm{du}$ trimestre précédent $X_{m 1(-1)}^{i}$ et $X_{m 1(-1)}^{i}-X_{m 3(-2)}^{i}$ ). Nous considérons seulement les valeurs courantes des soldes du mois 1 relatifs à l'activité passée $\left(P P A_{m 1}^{m a n}, S T O_{m 1}^{m a n}, C A P A_{m 1}^{s v}, R E P A_{Q}^{s v}, P P A_{m 1}^{b a t}\right.$, $E F P A_{m 1}^{b a t}$ et $\left.T U C_{m 1}^{b a t}\right)$ en niveau et en variation. Il semble en effet peu probable que les perspectives d'activité au mois 1 soient utilisables pour prévoir le trimestre précédent (cela permet en outre d'échapper à d'éventuels biais d'endogénéité car ces soldes pourraient être liés à l'activité du trimestre précédent).

\subsection{La sélection des variables explicatives}

En tenant compte des différentes transformations, nous disposons à chaque mois de 64 variables explicatives potentielles (sans compter les termes autorégressifs et la constante), parmi lesquelles 26 relatives à l'industrie, 14 aux services et 24 au bâtiment ${ }^{14}$. Nous considérons deux méthodes alternatives de réduction de l'information apportée par ces variables : la méthode de sélection automatique GETS par blocs de Hendry et Krolzig (2005) et la méthode de combinaison (Stock et Watson, 2004).

\section{- La méthode de sélection automatique GETS}

La méthode de base développée par Hoovers et Perez (1999) et étendue par Hendry et Krolzig (1999, 2001) consiste à considérer un modèle général intégrant toutes les variables explicatives et à le réduire progressivement en éliminant les variables non significatives, tout en s'assurant que les modèles obtenus satisfont un certain nombre de tests de spécification. Nous utilisons ici le test de normalité de Doornick et Hansen (1994), un test du multiplicateur de Lagrange d'autocorrélation d'ordre 4 des résidus (Godfrey, 1978), le test d'hétéroscédasticité quadratique entre les régresseurs (Nicholls et Pagan, 1983) et le test d'échec prédictif de Chow sur 50\% et $90 \%$ de la période d'estimation. Cette approche conduit à retenir un petit nombre de modèles entre lesquels on discriminera en utilisant des tests d'englobement et, si ce n'est pas suffisant, des critères d'information ${ }^{15}$.

Le nombre de variables explicatives envisagées est important ici : nous considérons 64 soldes en niveau ou en différence et autorisons par ailleurs jusqu'à quatre termes autorégressifs. Le

\footnotetext{
${ }^{14}$ Nous considérons en effet 14 soldes mensuels en niveau au mois en cours et ces 14 soldes en variation mensuelle sur les 3 derniers mois observés et 4 soldes trimestriels en niveau et en variation trimestrielle. Nous disposons donc au total de 64 variables pour construire les étalonnages des différents mois.

${ }^{15}$ On pourra se référer à Dubois et Michaux (2006) pour une présentation plus exhaustive de cet algorithme. Un programme conçu par les mêmes auteurs est disponible sous Scilab (Dubois et Michaux, 2008).
} 
nombre de variables explicatives possibles étant proche du nombre d'observations trimestrielles du PIB dont nous disposons, l'utilisation de la version classique de l'algorithme GETS n'est pas possible. La mise en œuvre de l'algorithme débute en effet avec l'estimation du modèle le plus général ce qui exige un nombre d'observations supérieur au nombre de variables explicatives, constante comprise. Par ailleurs, certains tests (le test d'hétéroscédasticité et les tests de Chow) ne peuvent pas être appliqués si le nombre d'observations est faible par rapport au nombre de variables explicatives. Par conséquent, nous avons appliqué la variante par blocs de la méthode GETS (Hendry et Krolzig, 2005).

Dans cette variante, on constitue $K_{1}$ groupes de variables par tirages au hasard sans remise parmi les $N$ variables disponibles. Nous choisissons ici $K_{1}$ comme le nombre minimum tel que le nombre de variables du bloc ne dépasse pas le nombre maximal de variables $N / K_{1}<T_{\max }$ (la taille des blocs diminuera donc au fur et à mesure des itérations). Nous appliquons à chacun de ces groupes l'algorithme GETS. Nous utilisons à ce niveau des tests de seuils élevés pour compenser la faible qualité d'ajustement et l'autocorrélation résiduelle pouvant résulter de l'omission possible de variables explicatives du fait du partage de l'ensemble des variables en sous-groupes. Nous conservons les variables retenues des $K_{1}$ groupes. La composition des $K_{1}$ groupes pouvant affecter le choix des variables, nous réitérons cette procédure $L$ fois ${ }^{16}$. A l'issue de ces calculs, si le nombre de variables sélectionnées au moins une fois $M_{1}$ dépasse encore le nombre maximal de variables, $M_{1}>T_{\max }$, nous constituons de nouveau $K_{2}$ blocs de variables par tirages au hasard sans remise parmi les $M_{1}$ variables et appliquons de nouveau à chacun d'eux l'algorithme GETS mais en utilisant des seuils moins élevés ${ }^{17}$. On répète ces $L$ tirages aléatoires jusqu'à ce que $M_{i}<T_{\max }$. On applique alors la méthode GETS classique aux $M_{i}$ variables retenues $^{18}$.

Notons que dans une dernière étape, certaines variables explicatives qui avaient un signe contre-intuitif et/ou étaient peu fiables au sens du critère de "fiabilité" de Hendry et Krolzig ont été retirées des modèles (le critère de fiabilité donne le pourcentage de fois qu'une variable est significative sur l'échantillon total, la première et la deuxième partie de l'échantillon). Nous avons vérifié dans ce cas que les modèles obtenus satisfaisaient toujours aux différents tests de spécification.

Cette méthode est appliquée pour chaque mois du trimestre et pour chaque horizon de prévision. On dispose ainsi de sept étalonnages différents suivant le mois auquel on se situe au moment de la prévision et suivant le trimestre que l'on cherche à prévoir. Trois étalonnages distincts sont ainsi utilisés au mois 1 (le premier pour prévoir le taux de croissance de l'activité au trimestre précédent, le deuxième pour le trimestre en cours et le troisième pour prévoir le trimestre suivant $)^{19}$ et deux étalonnages distincts aux mois 2 et 3 (permettant de prévoir la croissance de l'activité aux trimestres en cours et suivant) ${ }^{20}$.

\footnotetext{
${ }^{16}$ Dans notre application, la procédure est réitérée $L=10$ fois.

${ }^{17}$ A la première itération, les seuils des tests de significativité des coefficients s'élèvent à $10 \%$ et ceux des tests de spécification à $5 \%$. Nous réduisons ensuite les seuils de $1 \%$ à chaque nouvelle itération jusqu'à ce qu'ils atteignent les valeurs usuelles de la procédure classique de $5 \%$ et $1 \%$ respectivement qui sont recommandées par Hendry et Krolzig (2001).

${ }^{18}$ Nous utilisons alors les seuils préconisés par Hendry et Krolzig (2001) de 5\% pour les tests de significativité et de $1 \%$ pour les tests de spécification.

${ }^{19}$ Comme on l'a indiqué plus haut, le PIB du trimestre T n'est publié qu'au deuxième mois du trimestre suivant $\mathrm{T}+1$.

${ }^{20}$ Nous privilégions ici l'approche dite directe utilisant une équation par horizon, plutôt que la méthode consistant à obtenir les prévisions par itération d'une équation unique. L'approche directe a l'avantage de ne pas
} 


\section{- La méthode de combinaison}

Cette méthode consiste à agréger des prévisions issues de spécifications bivariées. La construction des prévisions suivant cette approche comporte les étapes suivantes :

a) Dans un premier temps, on estime pour une variable explicative $X_{t}^{i}$ donnée et pour un horizon de prévision $h^{21}$ :

$$
Y_{t+h}=\beta_{0}^{h}+\beta_{1}^{h} X_{t}^{i}+\ldots+\beta_{p}^{h} X_{t-p}^{i}+\phi_{1}^{h} Y_{t-1}+\ldots+\phi_{q}^{h} Y_{t-q}+u_{t}^{h}
$$

pour $t=1, \ldots, T-h-1$ (ou $t=1, \ldots, T-h-2$ au mois 1 ), où $Y_{t}=q_{t}-q_{t-1}$ et $q_{t}$ le logarithme du PIB réel (en prix chaînés).

Suivant Stock et Watson, on autorise de 0 à 4 retards pour $Y_{t}$ (l'équation pourra donc ne pas comporter de terme autorégressif) et de 0 à 3 retards pour $X_{t}$; les 20 combinaisons possibles sont estimées sur l'échantillon total et l'on retient la spécification minimisant le critère $\mathrm{BIC}^{22}$.

b) On en déduit une estimation de la série à la période courante $T$ et une prévision de la série au trimestre suivant $T+1$ :

$$
\begin{aligned}
& \hat{Y}_{T \mid T}=\hat{\beta}_{0}^{1}+\hat{\beta}_{1}^{1} X_{T}^{i}+\ldots+\hat{\beta}_{p}^{1} X_{T-p}^{i}+\hat{\phi}_{1}^{1} Y_{T-1}+\ldots+\hat{\phi}_{q}^{1} Y_{T-q} \\
& \hat{Y}_{T+1 \mid T}=\hat{\beta}_{0}^{2}+\hat{\beta}_{1}^{2} X_{T}^{i}+\ldots+\hat{\beta}_{p}^{2} X_{T-p}^{i}+\hat{\phi}_{1}^{2} Y_{T-1}+\ldots+\hat{\phi}_{q}^{2} Y_{T-q}
\end{aligned}
$$

Au mois 1, une estimation du taux de croissance du produit doit également être construite pour le trimestre précédent $T-1$ :

$$
\begin{aligned}
& \hat{Y}_{T-1 \mid T}=\hat{\beta}_{0}^{1}+\hat{\beta}_{1}^{1} X_{T}^{i}+\ldots+\hat{\beta}_{p}^{1} X_{T-p}^{i}+\hat{\phi}_{1}^{1} Y_{T-2}+\ldots+\hat{\phi}_{q}^{1} Y_{T-q-1} \\
& \hat{Y}_{T \mid T}=\hat{\beta}_{0}^{2}+\hat{\beta}_{1}^{2} X_{T}^{i}+\ldots+\hat{\beta}_{p}^{2} X_{T-p}^{i}+\hat{\phi}_{1}^{2} Y_{T-2}+\ldots+\hat{\phi}_{q}^{2} Y_{T-q-1} \\
& \hat{Y}_{T+1 \mid T}=\hat{\beta}_{0}^{3}+\hat{\beta}_{1}^{3} X_{T}^{i}+\ldots+\hat{\beta}_{p}^{3} X_{T-p}^{i}+\hat{\phi}_{1}^{3} Y_{T-2}+\ldots+\hat{\phi}_{q}^{3} Y_{T-q-1}
\end{aligned}
$$

c) La démarche est répliquée pour chaque variable explicative potentielle (seules les variables qui améliorent le critère BIC par rapport au processus autorégressif univarié sont retenues). On obtient alors un ensemble de prédicteurs pour les différents horizons qui sont ensuite combinés (en calculant au choix leur moyenne, leur moyenne tronquée, leur médiane ou leur moyenne pondérée en fonction des erreurs passées $)^{23,24}$.

Contrairement à la méthode de sélection automatique classique, cette approche a l'avantage de ne pas contraindre le nombre de variables explicatives. En effet, la méthode repose sur des étalonnages bivariés (autant que de variables explicatives envisagées).

nécessiter de modèles auxiliaires pour construire les prévisions des variables explicatives qui seraient alors nécessaires (voir par exemple Marcellino, Stock et Watson, 2006, pour une discussion sur ce point).

${ }^{21}$ De même, nous privilégions ici l'approche directe dans la construction de nos prévisions.

${ }^{22}$ L'utilisation du critère AIC conduisait à des prévisions de qualité similaire.

${ }^{23}$ Si l'on dispose de $n$ prédicteurs, le poids du prédicteur $i$ est alors donné par $w_{i t}=m_{i t}^{-1} / \sum_{j=1}^{n} m_{j t}^{-1}$ avec $m_{i t}=\sum_{s=T_{0}}^{t-h} \delta^{t-h-s}\left(Y_{s+h}-\hat{Y}_{i, s+h \mid s}\right)^{2}$ en utilisant les prévisions réalisées en $T_{0}, \ldots, t-h$. Le paramètre $\delta$ permet de pénaliser les erreurs de prévision les plus anciennes. Ainsi, si $\delta<1$, on accordera une importance plus importante aux prédicteurs les plus performants sur la période récente.

${ }^{24}$ Des techniques alternatives de pondération sont présentées par Stock et Watson (2004) et dans des études plus récentes comme celle de Altavilla et Cicarelli (2007). 


\section{Résultats d'estimation et qualité d'ajustement}

Les deux méthodes ont été appliquées sur la totalité de l'échantillon du deuxième trimestre de 1988 au quatrième trimestre de 2006. Nous présentons dans un premier temps les étalonnages retenus, puis comparons la qualité de l'ajustement du taux de croissance du PIB dans les deux méthodes.

\subsection{Les étalonnages retenus}

\section{- La méthode de sélection automatique GETS}

Les sept étalonnages construits avec l'algorithme GETS sont décrits dans le tableau 2a. Nous y présentons les variables retenues suivant le mois et l'horizon de prévision.

Tableau 2a. Variables sélectionnées par l'algorithme de sélection automatique

\begin{tabular}{|c|c|c|}
\hline MOIS & $\begin{array}{c}\text { TRIM. } \\
\text { PREVU } \\
\end{array}$ & MODELE \\
\hline \multirow{3}{*}{1} & $\mathrm{~T}-1$ & $\begin{array}{l}\text { Constante, } P P A_{m 1}^{\operatorname{man}}, C A P A_{m 1}^{s v}-C A P A_{m 3(-1)}^{s v}, E F P A_{m 1}^{b a t}, T U C_{m 1}^{b a t}, P P E R_{m 2(-1)}^{\operatorname{man}}, \\
P G E N_{m 1(-1)}^{\operatorname{man}}, P G E N_{m 2(-1)}^{\operatorname{man}}-P G E N_{m 1(-1)}^{\operatorname{man}}, D A_{Q(-1)}^{\operatorname{man}}-D A_{Q(-2)}^{\operatorname{man}}\end{array}$ \\
\hline & $\mathrm{T}$ & Constante, $P P E R_{m 1}^{m a n}, T U C_{m 1}^{b a t}, C A P A_{m 2(-1)}^{s v}-C A P A_{m 1(-1)}^{s v}, C C_{m 3(-1)}^{b a t}-C C_{m 2(-1)}^{b a t}$ \\
\hline & $\mathrm{T}+1$ & $\begin{array}{l}\text { Constante, } C A P A_{m 1}^{s v}, T U C_{m 1}^{b a t}, P P E R_{m 1}^{b a t}-P P E R_{m 3(-1)}^{b a t}, P P E R_{m 3(-1)}^{m a n}-P P E R_{m 2(-1)}^{m a n}, \\
C A P R E_{m 2(-1)}^{s v}-C A P R E_{m 1(-1)}^{s v}, T U C_{m 2(-1)}^{b a t}-T U C_{m 1(-1)}^{b a t}\end{array}$ \\
\hline \multirow{2}{*}{2} & $\mathrm{~T}$ & $\begin{array}{l}\text { Constante, } C C_{m 2}^{\operatorname{man}}, P P A_{m 2}^{\operatorname{man}}-P P A_{m 1}^{\operatorname{man}}, P P A_{m 1}^{\operatorname{man}}-P P A_{m 3(-1)}^{\operatorname{man}}, D A_{Q}^{\operatorname{man}}-D A_{Q(-1)}^{\operatorname{man}}, \\
C A P A_{m 3(-1)}^{s v}-C A P A_{m 2(-1)}^{s v}\end{array}$ \\
\hline & $\mathrm{T}+1$ & $\begin{array}{l}\text { Constante, } C C E_{m 2}^{\operatorname{man}}, P G E N_{m 2}^{\operatorname{man}}-P G E N_{m 1}^{m a n}, P P E R_{m 2}^{b a t}, T U C_{m 2}^{b a t}, D A_{Q}^{\text {man }}, R E P A_{Q}^{s v} \\
D A_{Q}^{\text {man }}-D A_{Q(-1)}^{\operatorname{man}}\end{array}$ \\
\hline \multirow[t]{2}{*}{3} & $\mathrm{~T}$ & $\begin{array}{l}\text { Constante, } C C_{m 3}^{m a n}, T U C_{m 3}^{b a t}-T U C_{m 2}^{b a t}, C A P A_{m 3}^{s v}-C A P A_{m 2}^{s v}, P P A_{m 1}^{m a n}-P P A_{m 3(-1)}^{m a n}, \\
D A_{Q}^{m a n}-D A_{Q(-1)}^{\operatorname{man}}, C A P R E_{m 1}^{s v}-C A P R E_{m 3(-1)}^{s v}, R E P A_{Q}^{s v}-R E P A_{Q(-1)}^{s v}, \\
E F P R E_{m 1}^{b a t}-E F P R E_{m 3(-1)}^{b a t}\end{array}$ \\
\hline & $\mathrm{T}+1$ & $\begin{array}{l}\text { Constante, } C C E_{m 3}^{\operatorname{man}}, C C_{m 3}^{\operatorname{man}}-C C_{m 2}^{m a n}, P G E N_{m 2}^{\operatorname{man}}-P G E N_{m 1}^{\operatorname{man}}, T U C_{m 2}^{b a t}-T U C_{m 1}^{b a t}, \\
D A_{Q}^{\operatorname{man}}, D A_{Q}^{\operatorname{man}}-D A_{Q(-1)}^{\operatorname{man}}, R E P A_{Q}^{s v}, C A P A_{m 2}^{s v}-C A P A_{m 1}^{s v}\end{array}$ \\
\hline
\end{tabular}

Les sept étalonnages comportent de quatre à huit variables explicatives et aucun terme autorégressif. Ce dernier résultat est intéressant, car il rend nos prévisions moins sensibles aux révisions de PIB. Rappelons en effet que le taux de croissance du PIB n'est définitif que trois ans après la première publication et qu'il est soumis auparavant à des variations qui peuvent être importantes. En l'absence de terme autorégressif dans l'équation, les prédicteurs seront moins sensibles à ces variations (voire pas du tout si les modèles sont estimés en retirant les trois dernières années de la période d'estimation, comme c'est souvent le cas en pratique).

Tous les étalonnages comportent par ailleurs plusieurs soldes du mois en cours, signe qu'il est utile de prendre en compte l'évolution de l'ensemble d'information au cours du trimestre. Les 
soldes interviennent à la fois en niveau et en variation. Tous les étalonnages comportent des soldes de l'enquête industrie mais ils incluent également des soldes des deux autres secteurs, à l'exception de l'étalonnage du mois 2 du trimestre courant sans solde de l'enquête dans le bâtiment. Les soldes relatifs aux services et au bâtiment constituent donc un complément utile à ceux fournis par l'enquête dans l'industrie (voir Darné et Brunhes-Lesage, 2007, ErkelRousse et Minodier, 2008 pour une étude plus approfondie de l'apport de l'enquête dans les services).

Pour illustrer ce dernier point, nous avons représenté en annexe 2 la contribution de chaque secteur à l'ajustement et calculé la contribution moyenne de chaque secteur sur la période considérée. La contribution d'un secteur à la variation estimée du taux de croissance en $t$ autour de sa moyenne est obtenue en sommant les soldes centrés du secteur en $t$ pondérés par leur coefficient estimé.

La contribution des soldes de l'enquête dans l'industrie à la série estimée domine pour la prévision du trimestre courant et précédent: les soldes dans l'industrie contribuent en moyenne aux différents mois à $61 \%$ aux fluctuations du taux de croissance autour de sa moyenne contre $19 \%$ pour les services et $20 \%$ pour le bâtiment (voir le tableau donné en annexe 2). En revanche, la répartition des contributions des trois enquêtes est plus équilibrée pour la prévision du taux de croissance de l'activité au trimestre suivant : la contribution de l'industrie n'est plus que de $33 \%$, tandis que celle des services s'élève à $39 \%$ et celle du bâtiment à $28 \%$. Ainsi, les soldes de l'enquête dans le bâtiment, rarement pris en compte dans les études, contribuent fortement à la prévision du trimestre $\mathrm{T}+1$.

\section{- La méthode de combinaison}

Le tableau $2 \mathrm{~b}$ présente les caractéristiques des étalonnages utilisés dans la méthode de combinaison: le nombre de soldes retenus des trois enquêtes et les soldes des trois enquêtes présentant le plus fort pouvoir explicatif mesuré par le $\mathrm{R}^{2}$ dans les étalonnages bivariés.

On note cette fois que les soldes de l'enquête dans le bâtiment sont le plus mobilisés, en particulier pour la prévision du trimestre suivant (on retrouve à ce niveau les observations faites à partir du graphique des contributions pour la méthode GETS). Les soldes de l'enquête dans les services interviennent moins souvent mais ils sont aussi moins nombreux dans l'ensemble de variables explicatives.

S'agissant des soldes présentant les meilleurs $\mathrm{R}^{2}$, on remarque le succès de l'activité prévue dans les services et des effectifs prévus dans le bâtiment et cela quels que soient le mois et l'horizon de prévision. Dans le cas de l'industrie, les résultats sont plus variés, divers soldes prospectifs, des perspectives générales aux perspectives personnelles de production et aux carnets de commandes rentrant dans les meilleurs étalonnages. La performance des perspectives générales de production dans l'industrie également sélectionnées dans trois des étalonnages construits avec la méthode GETS (cf. tableau 2a) n'était pas attendue, car les perspectives générales reflètent a priori davantage le climat ambiant tel qu'il est ressenti par les chefs d'entreprise qu'une réelle anticipation de la production. 
Tableau 2b. Variables sélectionnées dans la méthode de combinaison

\begin{tabular}{|c|c|c|c|c|c|c|c|}
\hline \multirow[t]{2}{*}{ MOIS } & \multirow[t]{2}{*}{$\begin{array}{c}\text { TRIM. } \\
\text { PREVU } \\
\end{array}$} & \multicolumn{2}{|r|}{ Industrie } & \multicolumn{2}{|r|}{ Services } & \multicolumn{2}{|r|}{ Bâtiment } \\
\hline & & $\mathrm{nb}$ & $\begin{array}{c}\text { Les } 3 \text { soldes de plus } \\
\text { fort } R^{2}\end{array}$ & $\mathrm{nb}$ & $\begin{array}{c}\text { Les } 3 \text { soldes de plus } \\
\text { fort } \mathrm{R}^{2}\end{array}$ & $\mathrm{nb}$ & $\begin{array}{c}\text { Les } 3 \text { soldes de plus } \\
\text { fort } \mathrm{R}^{2} \\
\end{array}$ \\
\hline \multirow{9}{*}{1} & & & $\triangle P G E N_{m 2(-1)}^{\operatorname{man}}$ & & $\triangle C A P R E_{m 2(-1)}^{s v}$ & & $E F P R E_{m 1(-1)}^{b a t}$ \\
\hline & $\mathrm{T}-1$ & 16 & $S T O_{m 1}^{\operatorname{man}}$ & 4 & $\Delta C A P A_{m 2(-1)}^{S v}$ & 16 & $P P A_{m 1}^{b a t}$ \\
\hline & & & $\triangle P P E R_{m 3(-1)}^{\operatorname{man}}$ & & $\triangle C A P R E_{m 3(-1)}^{s v}$ & & $\triangle P P E R_{m 1(-1)}^{b a t}$ \\
\hline & & & $\Delta C C_{m 1}^{\text {man }}, \Delta D A_{O}^{\text {man }}$ & & $\triangle C A P R E_{p \eta(-1)}^{s v}$ & & $E F P R E_{m 1}^{b a t}$ \\
\hline & $\mathrm{T}$ & 13 & $D A^{\operatorname{man}}$ & 2 & RFPASSV & 16 & $\triangle P P E R_{m 1}^{b a t}$ \\
\hline & & & & & КLI АD Q & & $\Delta E F P R E_{m 1}^{b a t}$ \\
\hline & & & $\Delta C C_{m 1}^{\operatorname{man}}$ & & & & $P P A_{m 1}^{b a t}$ \\
\hline & $\mathrm{T}+1$ & 4 & $\Delta C C E_{m 1}^{m a n}$ & 3 & 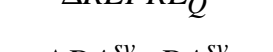 & 16 & $\Delta E F P R E_{m 1}^{b a t}$ \\
\hline & & & $\Delta S T O_{m 1}^{\operatorname{man}}$ & & $\Delta \boldsymbol{\Delta} \boldsymbol{A}_{Q}, \boldsymbol{D} \boldsymbol{A}_{Q}$ & & $E F P R E_{m 1}^{b a t}$ \\
\hline \multirow{4}{*}{2} & & & $\triangle P G E N_{m 2}^{\operatorname{man}}$ & & $\triangle C A P R E_{m 2}^{s v}$ & & $E F P R E_{m 2}^{b a t}$ \\
\hline & $\mathrm{T}$ & 11 & $P G E N_{m 2}^{\operatorname{man}}$ & 4 & $\triangle C A P A_{m 2}^{s v}$ & 15 & $P P E R_{m 2}^{b a t}$ \\
\hline & & & $\Delta D A_{Q}^{\operatorname{man}}$ & & $R E P A S_{Q}^{S V}$ & & $C C_{m 2}^{b a t}$ \\
\hline & $\mathrm{T}+1$ & 2 & $\begin{array}{c}\Delta P G E N_{m 2}^{\operatorname{man}} \\
\Delta C C_{m 1}^{\operatorname{man}}\end{array}$ & 1 & $\triangle C A P R E_{m 2}^{s v}$ & 14 & $\begin{array}{c}\Delta T U C_{m 2}^{b a t} \\
C C_{m 2}^{b a t}, E F P R E_{m 2}^{b a t}\end{array}$ \\
\hline \multirow{4}{*}{3} & & & $\triangle P G E N_{m 2}^{\operatorname{man}}$ & & $\triangle C A P R E_{m 2}^{s v}$ & & $E F P R E_{m 3}^{b a t}$ \\
\hline & $\mathrm{T}$ & 15 & $P P E R_{m 3}^{\operatorname{man}}$ & 6 & $C A P R E_{m 3}^{S v}$ & 17 & $P P E R_{m 3}^{b a t}$ \\
\hline & & & $P G E N_{m 3}^{\operatorname{man}}$ & & $\triangle C A P A_{m 2}^{s v}$ & & $\triangle P P E R_{m 1}^{b a t}$ \\
\hline & $\mathrm{T}+1$ & 7 & $\begin{array}{c}\Delta P G E N_{m 2}^{\operatorname{man}} \\
\Delta C C_{m 1}^{\operatorname{man}}, C C_{m 3}^{\operatorname{man}}\end{array}$ & 1 & $\triangle C A P R E_{m 2}^{S v}$ & 14 & $\begin{array}{c}\Delta T U C_{m 2}^{b a t} \\
C C_{m 3}^{b a t}, P P A_{m 3}^{b a t}\end{array}$ \\
\hline
\end{tabular}

Lecture : Le tableau reporte le nombre de soldes retenus dans chaque secteur et les trois soldes présentant le meilleur $\mathrm{R}^{2}$ pour chaque secteur.

\subsection{Analyse de la qualité d'ajustement des modèles}

Nous examinons à présent la qualité de l'ajustement du taux de croissance du PIB obtenu à partir des modèles décrits plus haut et par combinaison.

Le tableau 3 reporte les coefficients de détermination des étalonnages utilisés dans les deux méthodes. Dans la méthode de combinaison, nous disposons d'autant de coefficients de détermination que de variables retenues car nous ne disposons pas d'un modèle unique. Nous reportons donc dans ce cas les coefficients moyen, maximum et minimum, ainsi que le coefficient d'asymétrie de la série de coefficients.

Les modèles retenus dans la méthode de combinaison présentent en moyenne des coefficients de détermination très inférieurs à ceux des modèles construits avec l'algorithme GETS. La qualité d'ajustement des modèles dans les deux méthodes s'améliore par ailleurs à mesure que l'on intègre de l'information nouvelle au cours du trimestre. Dans la méthode de sélection automatique, le coefficient de détermination augmente ainsi de 0,56 au mois 1 du trimestre en cours à 0,76 au mois $1 \mathrm{du}$ trimestre suivant. Pour la prévision du trimestre suivant, le $\mathrm{R}^{2} \mathrm{du}$ 
modèle du mois 1 est de 0,53 et au mois 3 , il s'élève à 0,64 . La qualité d'ajustement s'améliore également au cours du temps dans la méthode de combinaison. Le coefficient moyen augmente ainsi de 0,27 à 0,32 pour la prévision du trimestre courant ou précédent et de 0,18 à 0,27 pour la prévision du trimestre suivant entre le mois 1 et le mois 2 . On note toutefois une légère dégradation du coefficient de détermination moyen au mois 3 . On constate également généralement une progression des coefficients maximum et minimum ${ }^{25}$.

Dans la méthode de combinaison, on notera également que les coefficients d'asymétrie des coefficients de détermination et des coefficients de détermination ajustés sont positifs, ce qui signifie que la distribution des coefficients de détermination est étalée vers la droite où les coefficients de détermination sont plus élevés (les plus grandes valeurs ont une fréquence d'apparition plus élevée que les petites valeurs).

Tableau 3. Qualité d'ajustement des modèles

\begin{tabular}{|c|c|c|c|c|c|c|c|c|}
\hline \multirow{2}{*}{ Méthode } & $\begin{array}{c}\text { Trimestre } \\
\text { prévu }\end{array}$ & \multirow{2}{*}{ T-1 } & \multicolumn{3}{|c|}{ T } & \multicolumn{3}{c|}{ T+1 } \\
\cline { 2 - 10 } & Mois & M1 & M1 & M2 & M3 & M1 & M2 & M3 \\
\hline GETS & $R^{\mathbf{2}}$ & 0,76 & 0,56 & 0,69 & 0,74 & 0,53 & 0,61 & 0,64 \\
\hline COMB & $R^{\mathbf{2}}$ moy & 0,32 & 0,27 & 0,31 & 0,32 & 0,18 & 0,27 & 0,25 \\
\hline COMB & $R^{\mathbf{2}} \max$ & 0,47 & 0,39 & 0,47 & 0,47 & 0,28 & 0,34 & 0,34 \\
\hline COMB & $R^{\mathbf{2}} \min$ & 0,24 & 0,19 & 0,24 & 0,25 & 0,11 & 0,20 & 0,20 \\
\hline COMB & Skewness & 1,11 & 0,53 & 1,41 & 1,09 & 0,21 & 0,29 & 0,81 \\
\hline GETS & $\bar{R}^{2}$ & 0,73 & 0,53 & 0,67 & 0,69 & 0,48 & 0,57 & 0,59 \\
\hline COMB & $\bar{R}^{2}$ moy & 0,29 & 0,25 & 0,28 & 0,29 & 0,15 & 0,24 & 0,22 \\
\hline COMB & $\bar{R}^{2}$ max & 0,41 & 0,35 & 0,41 & 0,41 & 0,26 & 0,30 & 0,30 \\
\hline COMB & $\bar{R}^{2}$ min & 0,22 & 0,17 & 0,22 & 0,23 & 0,08 & 0,18 & 0,18 \\
\hline COMB & Skewness & 1,02 & 0,53 & 1,19 & 1,06 & 0,20 & 0,02 & 0,57 \\
\hline
\end{tabular}

Lecture: La première partie du tableau reporte pour la méthode avec sélection automatique des variables (GETS) le coefficient de détermination $R^{2}$ et pour la méthode de combinaison $(C O M B)$, le coefficient de détermination moyen $R^{2}$ moy, le coefficient maximum $R^{2}$ max, le coefficient minimum $R^{2}$ min et le coefficient d'asymétrie de la série des $R^{2}$. Des calculs analogues sont effectués pour le coefficient de détermination ajusté dans la deuxième partie du tableau.

La figure 1 représente le taux de croissance estimé grâce aux modèles utilisés au mois 3 pour prévoir le taux de croissance du PIB au trimestre courant $\mathrm{T}^{26}$. Dans la méthode de combinaison, nous reportons uniquement l'ajustement moyen ${ }^{27}$. Sans surprise au regard des résultats précédents, la dynamique du PIB est mal retracée par la méthode de combinaison. Elle permet tout juste de capter la tendance de la série et parfois avec retard. Les reculs du PIB ne sont jamais reproduits. En revanche, la qualité d'ajustement paraît assez bonne sur les deux dernières décennies avec les modèles construits en utilisant l'algorithme GETS. En particulier, nos modèles rendent bien compte de l'épisode de récession en 1992-93 et du ralentissement de l'activité en 2001.

\footnotetext{
${ }^{25}$ Des observations analogues sont tirées des coefficients de détermination ajustés.

${ }^{26}$ Nous avons omis les résultats obtenus aux autres mois car ils sont assez proches.

${ }^{27}$ Les résultats sont similaires avec les trois autres techniques de pondération.
} 
Figure 1. Ajustement au mois 3 du taux de croissance du PIB au trimestre courant $T$

Ajustement du trimestre courant au mois 3 - méthode de combinaison

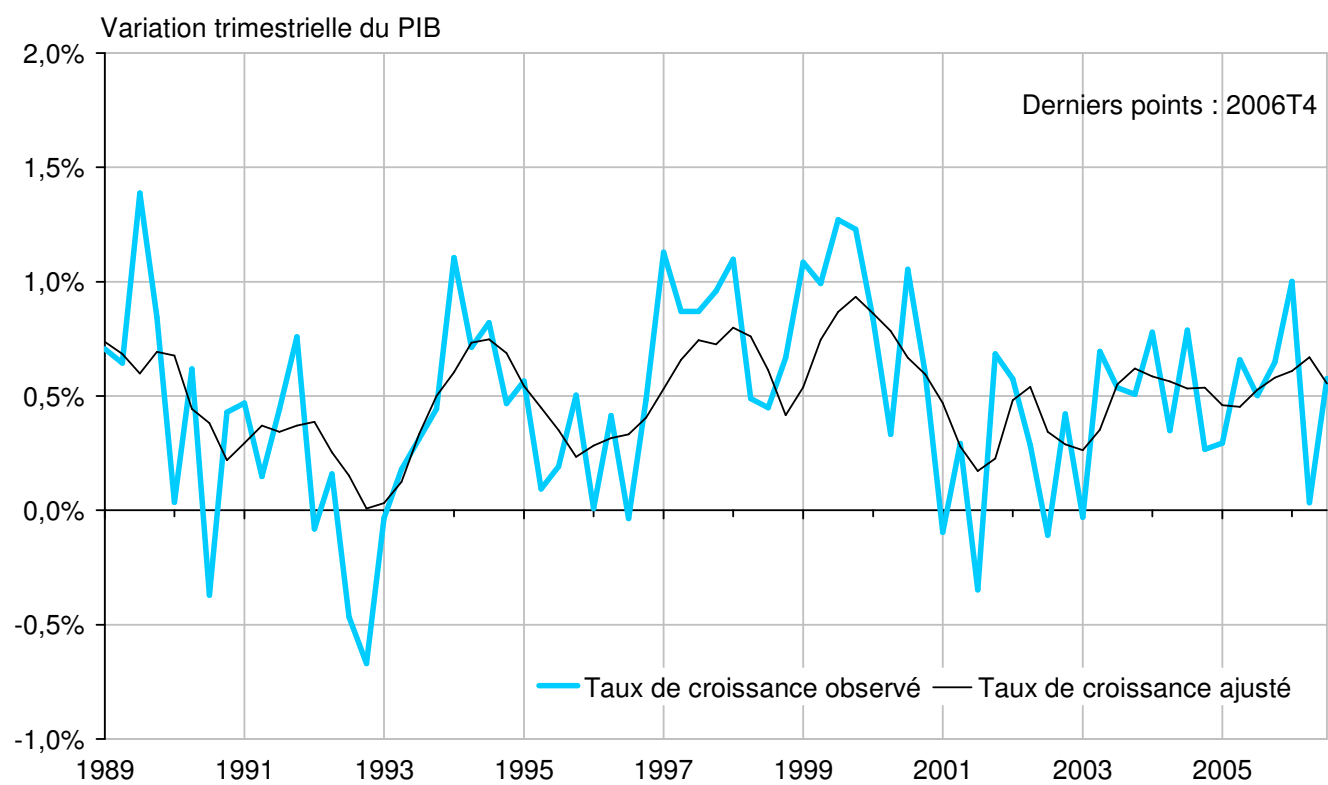

Ajustement du trimestre courant au mois 3 - méthode GETS

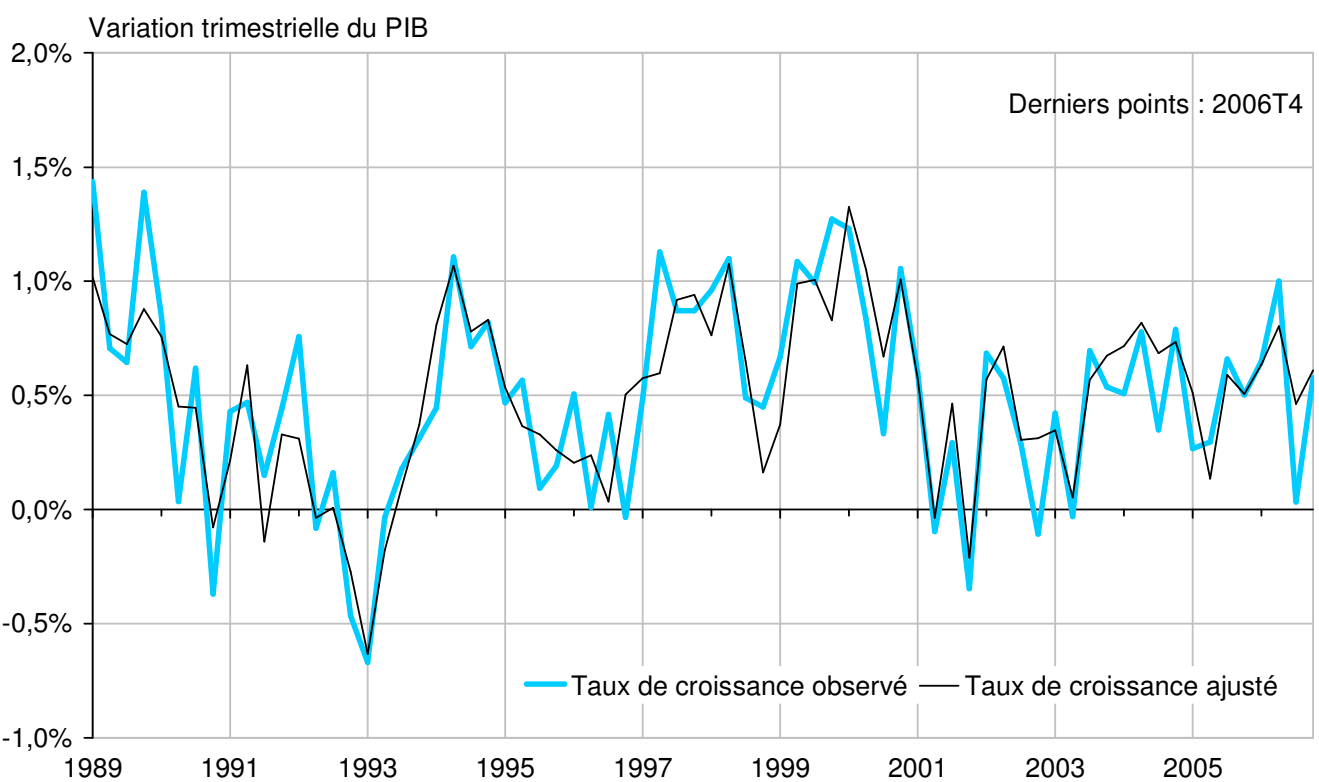




\section{Evaluation hors-échantillon : résultats de régressions récursives et roulantes}

Les performances des deux techniques de prévision sont maintenant évaluées horséchantillon, c'est-à-dire en dehors de la période d'estimation.

\subsection{Le cadre expérimental}

Pour juger des deux techniques de prévision présentées, nous nous plaçons dans des conditions réelles de prévision en faisant des évaluations hors échantillon, c'est-à-dire hors de la période d'estimation. Plutôt que de raisonner en échantillon, c'est-à-dire à partir des résidus estimés des modèles qui sont calculés à partir de l'information disponible avant, en l'instant et après l'instant que l'on souhaite prévoir, on se fonde sur les erreurs de prévision calculées seulement à partir de l'information disponible au moment de la prévision. Il est important de procéder de la sorte car les résultats peuvent différer sensiblement en échantillon et hors échantillon. Il faut donc privilégier l'évaluation hors échantillon qui correspond aux conditions réelles d'exercice du prévisionniste ${ }^{28,29}$.

A cette fin, on construit des prévisions récursives ou roulantes. On tronque notre échantillon de 75 observations des 21 dernières, on estime le ou les modèles suivant la technique de prévision utilisée sur la période restante, du deuxième trimestre de 1988 au deuxième trimestre de 2001, puis on construit les prévisions à différents pas et les erreurs de prévision associées. On augmente ensuite l'échantillon d'une observation, on ré-estime les modèles, on calcule les nouvelles prévisions et les erreurs de prévision correspondantes. On augmente ensuite de nouveau l'échantillon d'une observation, on réplique les calculs précédents et ainsi de suite jusqu'à ce que l'on ait balayé les 21 dernières observations. Dans le cas récursif, la date de la première observation est fixe de sorte que la taille de l'échantillon augmente progressivement. Dans le cas roulant, l'échantillon, cette fois-ci de taille fixe, roule.

Nous avons considéré ces deux configurations - roulante et récursive - pour mettre en évidence un éventuel effet de la taille de l'échantillon sur les performances des techniques de prévision. Si les relations estimées sont stables, on s'attend à ce que l'utilisation de toute l'information disponible au travers des régressions récursives conduise à de meilleures prévisions. Si les données sont hétérogènes ou en cas de changements structurels sur la période étudiée, les régressions roulantes réalisées sur de petits échantillons sont préférables (Clark et McCracken, 2004, Giacomini et White, 2006). En contrepartie, l'imprécision des estimateurs peut augmenter, puisque l'on utilise moins d'information dans l'estimation du modèle.

A l'issue de cette expérience, on dispose pour chaque technique de prévision évaluée d'une série d'erreurs de prévision auxquelles sont appliqués les critères d'évaluation classiques : les critères RMSFE, MAFE, le test de signe de Diebold et Mariano (1995) et un "test de zone". Les deux premiers critères mesurent la dispersion des erreurs de prévision et leur amplitude moyenne. Les deux tests permettent d'évaluer l'aptitude des modèles à prévoir le sens de variation (i.e. les hausses ou les baisses du taux de croissance de l'activité) et à donner à

\footnotetext{
${ }^{28}$ Notons toutefois que l'exercice n'est pas totalement hors échantillon car la sélection des variables explicatives dans la méthode GETS comme dans la méthode de combinaison décrite dans la section précédente a été réalisée en utilisant l'échantillon total.

${ }^{29}$ En dépit de ces limites, nous avons également reporté les résultats en échantillon en annexe 3 car ils ont l'avantage de se référer à une période plus longue (64 points au lieu de 21 points).
} 
raison des prévisions dans les zones $[-\infty, 0,5]$ et $[0,5,+\infty]$, soit au-dessous ou au-dessus du taux de croissance trimestriel moyen de l'économie française ${ }^{30}$.

Afin de mesurer l'apport des données d'enquête pour la prévision de court terme du taux de croissance de l'activité, les prévisions produites seront comparées à celles d'un processus autorégressif fondé sur la seule dynamique passée du taux de croissance de l'activité. S'agissant de l'ordre du processus, un processus AR d'ordre fixé à 4 a d'abord été retenu au vu des corrélogrammes, des corrélogrammes partiels et des tests de diagnostic usuels sur l'ensemble de la période (par analogie avec les deux autres techniques de prévision pour lesquelles la sélection des variables explicatives s'effectue sur l'ensemble de la période). Nous avons également considéré un processus d'ordre variable compris entre un et quatre et minimisant le critère $\mathrm{AIC}^{31}$ dans les régressions récursives ou roulantes. D'autres choix possibles pour la spécification de référence, parmi lesquels des processus autorégressifs à paramètres variables ou des spécifications non-linéaires à seuil sont discutés par Marcellino (2008) mais n'ont pas été testés ici ${ }^{32}$.

Enfin, compte tenu des différences importantes qui peuvent exister entre les premières publications du PIB, i.e. les chiffres publiés deux mois après la fin du trimestre concerné et les chiffres définitifs disponibles trois ans après, nous réalisons cet exercice à partir des erreurs de prévision des premiers résultats et des erreurs de prévision du taux de croissance du PIB publié début 2009. Il est intéressant d'évaluer la capacité des modèles à prévoir aussi les premières données publiées par l'Insee car les autorités gouvernementales ou monétaires peuvent être amenées à définir leurs politiques à partir des premières publications. Il ne s'agit néanmoins pas d'un exercice en temps réel, car nous utilisons les modèles estimés à partir des comptes définitifs. Pour les modèles ne comportant pas de termes autorégressifs, nos résultats doivent cependant se rapprocher de ceux d'un exercice en temps réel, puisque les données d'enquête sont peu révisées et puisqu'en pratique, les conjoncturistes écartent généralement les observations des trois dernières années pour estimer leur modèle.

\subsection{Les résultats}

Les résultats des prévisions récursives et roulantes réalisées aux différents mois du trimestre sur la période 2001T3-2006T4 sont reportés dans les tableaux $4^{33,34}$. Le tableau 4a reporte les critères d'évaluation calculés sur les données définitives de PIB, tandis que le tableau 4b présente ces mêmes critères obtenus sur les premiers résultats.

\footnotetext{
${ }^{30}$ Il s'agit d'un test unilatéral de proportion similaire dans sa construction à celui du test de signe de Diebold et Mariano.

${ }^{31}$ Le critère BIC conduisait à des résultats similaires.

${ }^{32}$ Pour la prévision du taux de croissance trimestriel du PIB américain de 1980 à 2004, Marcellino montre qu'il n'y a pas de gain à l'utilisation de spécifications autorégressives plus sophistiquées (modèle à seuil à transition lisse LSTAR, modèle à réseaux de neurones, modèles avec paramètres variables) mais indique qu'il est essentiel de spécifier correctement le processus linéaire.

${ }^{33}$ Dans la méthode de Stock et Watson, nous choisissons une valeur de 0,95 pour le paramètre $\delta$ intervenant dans le calcul de la moyenne pondérée en fonction des erreurs passées (ainsi, nous accordons un poids plus important aux prédicteurs les plus performants sur la période récente). Lorsque nous utilisons la moyenne tronquée, nous excluons $5 \%$ des prévisions les plus petites et $5 \%$ des prévisions les plus élevées.

${ }^{34}$ Les résultats en échantillon reportés en annexe 3 sont généralement cohérents ici avec ceux de l'évaluation hors échantillon mais on rappelle que les résultats hors échantillon doivent être privilégiés.
} 
Plusieurs enseignements se dégagent du tableau 4a. Les résultats obtenus sur les données définitives sont tout d'abord favorables à l'utilisation des données d'enquête pour prévoir le taux de croissance du PIB français, puisque les deux méthodes permettent toujours de gagner par rapport à un processus autorégressif univarié (la spécification avec sélection endogène du retard paraît à ce niveau préférable pour la prévision à un trimestre mais il y a peu de différences avec le processus autorégressif d'ordre fixe pour la prévision du trimestre courant ou précédent). Ainsi le gain en termes de RMSFE atteint $43 \%$ pour prévoir le trimestre courant $\mathrm{T}$. Les différences sont beaucoup moins sensibles pour le trimestre suivant $\mathrm{T}+1$ mais à certains mois, le gain peut tout de même atteindre $21 \%$. Nous retrouvons à ce niveau les résultats de Sédillot et Pain (2005) et de Erkel-Rousse et Minodier (2008) ${ }^{36}$.

Nos résultats conduisent par ailleurs à préconiser l'utilisation de la méthode de sélection automatique. Les critères RMSFE et MAFE sont toujours plus petits quand on utilise cette technique plutôt que la méthode de combinaison pour la prévision du trimestre précédent et courant. Pour la prévision du trimestre suivant, les deux méthodes donnent des résultats comparables $^{37}$. On notera également qu'à tous les horizons de prévision, les différences se creusent à mesure que l'on progresse dans le trimestre. Les résultats du test de signe et du test de zone donnent également l'avantage à la méthode GETS. La méthode de sélection automatique permet toujours de mieux détecter la localisation de la série par rapport à la moyenne de long terme que le hasard (en tirant à pile ou face) et cela quel que soit l'horizon considéré, contrairement à la méthode de combinaison. Par ailleurs, la méthode de Stock et Watson ne permet généralement pas de mieux détecter le sens de variation de la série que le hasard, alors que la première méthode fait toujours mieux pour le trimestre courant et précédent.

On retrouve également l'amélioration de la qualité des prévisions à mesure que l'on progresse dans le trimestre pour les étalonnages construits à partir de la méthode GETS. Ainsi, les critères RMSFE et MAFE sont plus petits pour les prévisions faites au mois 1 pour le trimestre précédent T-1 que ceux des prévisions du mois 3, puis des mois 2 et 1 du trimestre courant T. De même, on fait mieux au mois 3, puis au mois 2 et enfin au mois 1 pour prévoir le trimestre suivant $T+1^{38}$. Notons toutefois que l'essentiel de l'amélioration se fait entre le mois 1 et le mois 3 pour la prévision du trimestre $\mathrm{T}$ et qu'ensuite, l'amélioration est marginale. En revanche, il n'y a pas d'amélioration notable pour les prévisions issues de la méthode de combinaison ce qui renforce donc la supériorité de l'approche à une équation lorsque l'ensemble d'information sur le trimestre augmente ${ }^{39}$.

\footnotetext{
35 En principe, il conviendrait de tester si les critères RMSFE et MAFE obtenus suivant les différentes techniques de prévisions sont significativement différents. Il serait en particulier intéressant de comparer les critères obtenus en prenant en compte les soldes d'opinion aux critères obtenus sur les prévisions d'un processus AR. Comme Stock et Watson (2004) l'indiquent, cela pose problème pour la méthode de combinaison car les modèles sont parfois emboîtés, parfois non et la distribution des statistiques de test n'est pas connue dans ce cas.

${ }^{36}$ D'après Sédillot et Pain (2005), cette caractéristique est particulièrement forte en France ; dans la plupart des pays qu'ils considèrent, des modèles basés sur des données "dures" (production industrielle, consommation de produits manufacturés, immatriculations d'automobiles, etc) s'avèrent suffisants pour prévoir le taux de croissance du PIB au trimestre courant.

${ }^{37}$ Les résultats sont encore plus favorables sur l'échantillon total à la méthode GETS où les résultats sont meilleurs quel que soit l'horizon considéré (cf. annexe 3) mais l'exercice n'est plus hors échantillon dans ce cas.

${ }^{38}$ Cette amélioration de la qualité des prévisions à mesure que l'on progresse dans le trimestre a déjà été décrite dans la littérature à l'image de Dubois et Michaux (2006) pour la production manufacturière française, ErkelRousse et Minodier (2008) pour le PIB français, Sédillot et Pain (2005) pour le PIB des principales économies de l'OCDE dont la France, Drechsel et Maurin (2008) pour le PIB de la zone euro.

${ }^{39}$ Un résultat analogue est obtenu pour le taux de croissance du PIB de la zone euro par Angelini et al. (2008).
} 
On remarquera aussi que les prévisions obtenues dans les régressions roulantes ne sont pas sensiblement différentes de celles issues des régressions récursives. On trouve seulement une légère amélioration des résultats pour la méthode GETS dans les régressions roulantes pour la prévision du trimestre suivant. Le nombre d'observations à notre disposition est néanmoins vraisemblablement insuffisant pour dégager ici un effet de la taille de l'échantillon. Dans la méthode de combinaison, les différentes techniques de pondération des prédicteurs moyenne, moyenne tronquée, médiane et moyenne pondérée en fonction des erreurs de prévision passées - donnent également des résultats comparables. Des techniques de pondération simples donnent donc des résultats peu différents de techniques plus sophistiquées ${ }^{40}$.

La comparaison des prévisions aux premiers résultats des comptes trimestriels (tableau 4b) donne une image moins favorable de la méthode de sélection automatique. $\mathrm{Vu}$ des critères RMSFE et MAFE, on gagne cette fois à utiliser la méthode de combinaison plutôt que l'algorithme GETS à l'horizon $\mathrm{T}+1$. On ne retrouve par ailleurs plus systématiquement l'amélioration des critères d'un mois à l'autre du trimestre avec la méthode GETS. En revanche, les deux méthodes donnent des résultats comparables pour le test de zone et la méthode de sélection automatique permet de mieux prévoir le sens de variation de la série au trimestre courant et précédent. Rappelons néanmoins que ces derniers résultats doivent être pris avec beaucoup de précaution car si les étalonnages construits avec la méthode GETS généralement sans termes autorégressifs donnent une bonne image des résultats obtenus en temps réel, ceux présentés pour la méthode de combinaison en sont vraisemblablement plus éloignés car les étalonnages bivariés utilisés incluent la variable endogène retardée construite à partir des données actuelles du PIB.

Pour illustrer ces différentes observations, nous reportons enfin sur la figure 2 les prévisions récursives obtenues hors échantillon au mois 3 du taux de croissance courant avec les deux techniques de prévision concurrentes. Les prévisions du trimestre suivant $\mathrm{T}+1$ sont représentées en annexe 4.

\footnotetext{
${ }^{40}$ Dans l'étude de Stock et Watson (2004), les techniques de pondération conduisant aux meilleures prévisions des taux de croissance du PIB de 7 pays de l'OCDE pour des horizons de prévision allant de 2 à 8 trimestres sont les poids variables obtenus par le filtre de Kalman, la moyenne puis la moyenne tronquée, mais les résultats obtenus suivant ces trois approches sont également très proches.
} 
Figure 2. Prévisions hors-échantillon du trimestre courant T

Prévision du trimestre courant au mois 3 - méthode de combinaison

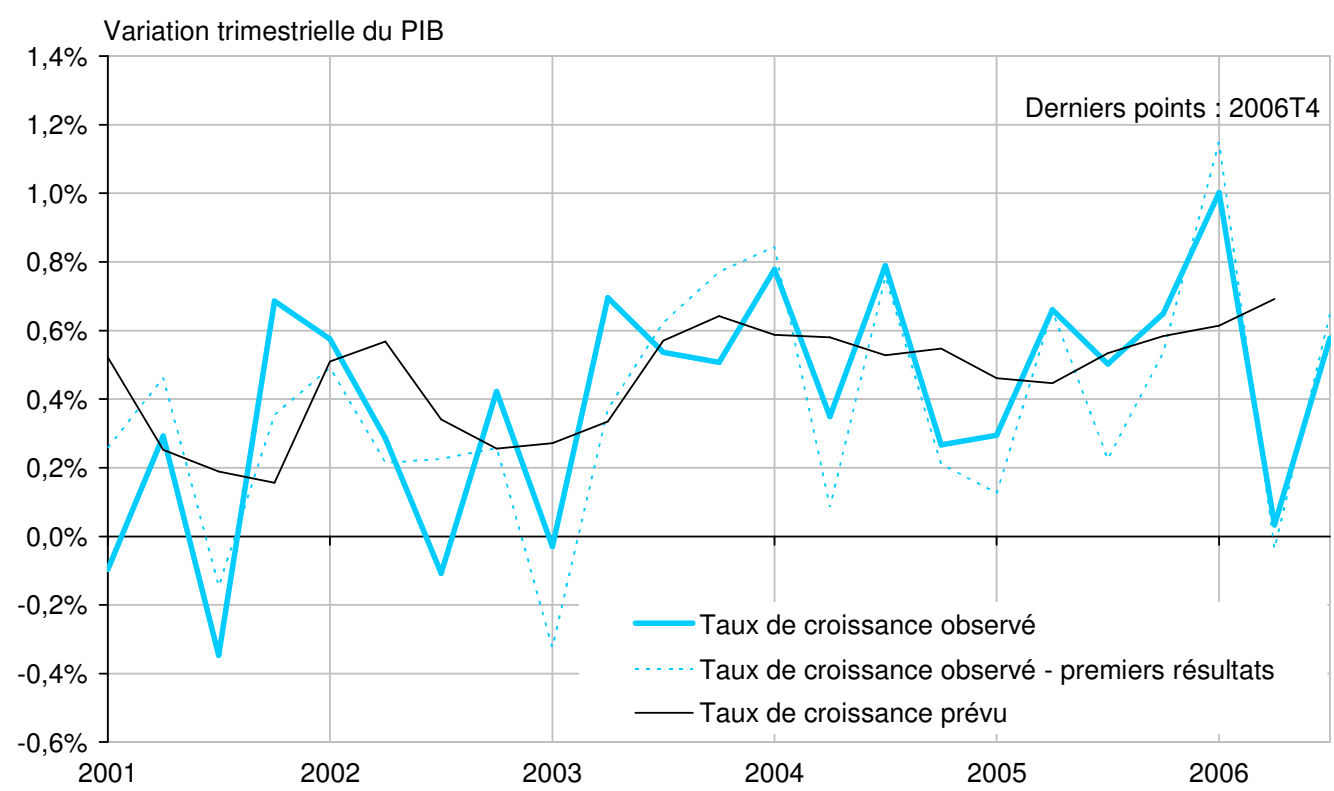

Prévision du trimestre courant au mois 3 - méthode GETS

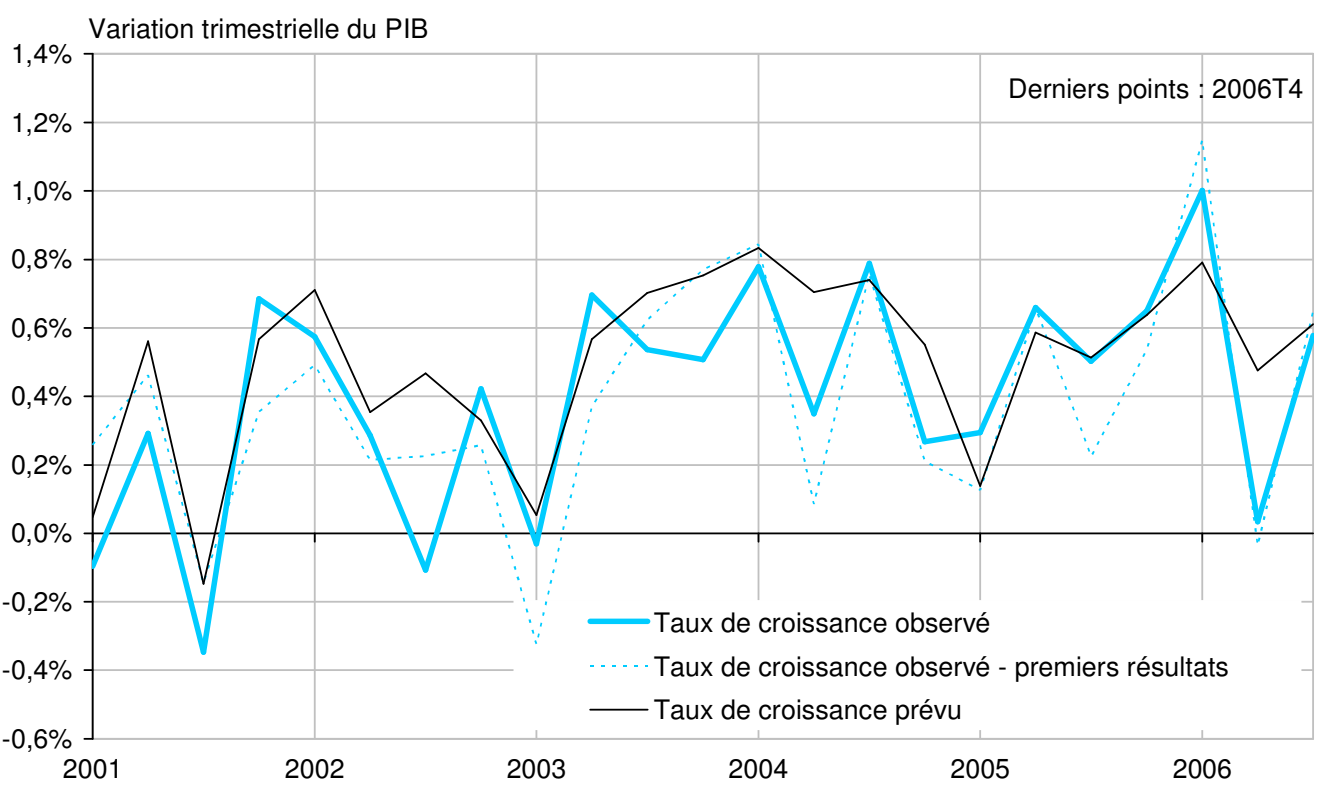


Tableau 4a. Résultats des prévisions récursives et roulantes - Données définitives

\begin{tabular}{|c|c|c|c|c|c|c|c|c|c|c|c|c|c|c|}
\hline \multirow{3}{*}{ RMSFE } & \multicolumn{7}{|c|}{ RECURSIF } & \multicolumn{7}{|c|}{ ROULANT } \\
\hline & T-1 & \multicolumn{3}{|c|}{$\mathbf{T}$} & \multicolumn{3}{|c|}{$\mathbf{T}+1$} & T-1 & \multicolumn{3}{|c|}{$\mathbf{T}$} & \multicolumn{3}{|c|}{$\mathbf{T}+1$} \\
\hline & Mois 1 & Mois 1 & Mois 2 & Mois 3 & Mois 1 & Mois 2 & Mois 3 & Mois 1 & Mois 1 & Mois 2 & Mois 3 & Mois 1 & Mois 2 & Mois 3 \\
\hline AR4 & $0,37 \%$ & $0,37 \%$ & $0,37 \%$ & $0,37 \%$ & $0,36 \%$ & $0,36 \%$ & $0,36 \%$ & $0,37 \%$ & $0,37 \%$ & $0,37 \%$ & $0,37 \%$ & $0,37 \%$ & $0,37 \%$ & $0,37 \%$ \\
\hline AR_AIC & $0,37 \%$ & $0,37 \%$ & $0,37 \%$ & $0,37 \%$ & $0,32 \%$ & $0,32 \%$ & $0,32 \%$ & $0,38 \%$ & $0,38 \%$ & $0,38 \%$ & $0,38 \%$ & $0,33 \%$ & $0,33 \%$ & $0,33 \%$ \\
\hline GETS & $0,19 \%$ & $0,27 \%$ & $0,24 \%$ & $0,21 \%$ & $0,37 \%$ & $0,34 \%$ & $0,28 \%$ & $0,19 \%$ & $0,27 \%$ & $0,24 \%$ & $0,20 \%$ & $0,37 \%$ & $0,32 \%$ & $0,26 \%$ \\
\hline Combm & $0,31 \%$ & $0,30 \%$ & $0,31 \%$ & $0,31 \%$ & $0,31 \%$ & $0,29 \%$ & $0,30 \%$ & $0,31 \%$ & $0,29 \%$ & $0,31 \%$ & $0,31 \%$ & $0,31 \%$ & $0,29 \%$ & $0,30 \%$ \\
\hline Combmt & $0,31 \%$ & $0,30 \%$ & $0,32 \%$ & $0,31 \%$ & $0,32 \%$ & $0,29 \%$ & $0,30 \%$ & $0,31 \%$ & $0,30 \%$ & $0,32 \%$ & $0,31 \%$ & $0,31 \%$ & $0,29 \%$ & $0,30 \%$ \\
\hline Combme & $0,31 \%$ & $0,30 \%$ & $0,32 \%$ & $0,32 \%$ & $0,32 \%$ & $0,29 \%$ & $0,31 \%$ & $0,31 \%$ & $0,30 \%$ & $0,32 \%$ & $0,32 \%$ & $0,32 \%$ & $0,29 \%$ & $0,30 \%$ \\
\hline Combfd & $0,31 \%$ & $0,29 \%$ & $0,31 \%$ & $0,31 \%$ & $0,31 \%$ & $0,29 \%$ & $0,28 \%$ & $0,31 \%$ & $0,29 \%$ & $0,31 \%$ & $0,30 \%$ & $0,30 \%$ & $0,28 \%$ & $0,28 \%$ \\
\hline \multirow{2}{*}{ MAFE } & T-1 & \multicolumn{3}{|c|}{$\mathbf{T}$} & \multicolumn{3}{|c|}{$\mathbf{T}+1$} & T-1 & \multicolumn{3}{|c|}{$\mathbf{T}$} & \multicolumn{3}{|c|}{$\mathbf{T}+1$} \\
\hline & Mois 1 & Mois 1 & Mois 2 & Mois 3 & Mois 1 & Mois 2 & Mois 3 & Mois 1 & Mois 1 & Mois 2 & Mois 3 & Mois 1 & Mois 2 & Mois 3 \\
\hline AR4 & $0,29 \%$ & $0,29 \%$ & $0,29 \%$ & $0,29 \%$ & $0,30 \%$ & $0,30 \%$ & $0,30 \%$ & $0,30 \%$ & $0,30 \%$ & $0,30 \%$ & $0,30 \%$ & $0,30 \%$ & $0,30 \%$ & $0,30 \%$ \\
\hline AR_AIC & $0,30 \%$ & $0,30 \%$ & $0,30 \%$ & $0,30 \%$ & $0,28 \%$ & $0,28 \%$ & $0,28 \%$ & $0,31 \%$ & $0,31 \%$ & $0,31 \%$ & $0,31 \%$ & $0,29 \%$ & $0,29 \%$ & $0,29 \%$ \\
\hline GETS & $0,16 \%$ & $0,23 \%$ & $0,20 \%$ & $0,16 \%$ & $0,26 \%$ & $0,25 \%$ & $0,22 \%$ & $0,16 \%$ & $0,23 \%$ & $0,19 \%$ & $0,16 \%$ & $0,26 \%$ & $0,24 \%$ & $0,21 \%$ \\
\hline Combm & $0,26 \%$ & $0,25 \%$ & $0,26 \%$ & $0,25 \%$ & $0,25 \%$ & $0,25 \%$ & $0,26 \%$ & $0,25 \%$ & $0,25 \%$ & $0,26 \%$ & $0,25 \%$ & $0,25 \%$ & $0,25 \%$ & $0,26 \%$ \\
\hline Combmt & $0,26 \%$ & $0,25 \%$ & $0,26 \%$ & $0,26 \%$ & $0,25 \%$ & $0,25 \%$ & $0,26 \%$ & $0,26 \%$ & $0,25 \%$ & $0,26 \%$ & $0,26 \%$ & $0,25 \%$ & $0,25 \%$ & $0,26 \%$ \\
\hline Combme & $0,26 \%$ & $0,25 \%$ & $0,27 \%$ & $0,26 \%$ & $0,26 \%$ & $0,25 \%$ & $0,26 \%$ & $0,26 \%$ & $0,25 \%$ & $0,26 \%$ & $0,26 \%$ & $0,26 \%$ & $0,25 \%$ & $0,26 \%$ \\
\hline Combfd & $0,25 \%$ & $0,24 \%$ & $0,26 \%$ & $0,26 \%$ & $0,25 \%$ & $0,24 \%$ & $0,23 \%$ & $0,25 \%$ & $0,24 \%$ & $0,26 \%$ & $0,25 \%$ & $0,24 \%$ & $0,24 \%$ & $0,24 \%$ \\
\hline \multirow{2}{*}{ Signe } & T-1 & \multicolumn{3}{|c|}{$\mathbf{T}$} & \multicolumn{3}{|c|}{$\mathbf{T}+\mathbf{1}$} & T-1 & \multicolumn{3}{|c|}{$\mathbf{T}$} & \multicolumn{3}{|c|}{$\mathbf{T + 1}$} \\
\hline & Mois 1 & Mois 1 & Mois 2 & Mois 3 & Mois 1 & Mois 2 & Mois 3 & Mois 1 & Mois 1 & Mois 2 & Mois 3 & Mois 1 & Mois 2 & Mois 3 \\
\hline AR4 & 0,67 & 0,67 & 0,67 & 0,67 & 0,19 & 0,19 & 0,19 & 0,96 & 0,96 & 0,96 & 0,96 & 0,19 & 0,19 & 0,19 \\
\hline AR_AIC & 0,99 & 0,99 & 0,99 & 0,99 & 0,04 & 0,04 & 0,04 & 0,99 & 0,99 & 0,99 & 0,99 & 0,19 & 0,19 & 0,19 \\
\hline GETS & 0,04 & 0,01 & 0,04 & 0,04 & 0,19 & 0,33 & 0,01 & 0,09 & 0,01 & 0,04 & 0,04 & 0,33 & 0,04 & 0,04 \\
\hline Combm & 0,96 & 0,19 & 0,96 & 0,96 & 0,50 & 0,04 & 0,04 & 0,91 & 0,19 & 0,96 & 0,91 & 0,33 & 0,04 & 0,09 \\
\hline Combmt & 0,96 & 0,19 & 0,99 & 0,96 & 0,50 & 0,01 & 0,04 & 0,91 & 0,33 & 0,99 & 0,99 & 0,50 & 0,01 & 0,09 \\
\hline Combme & 0,96 & 0,33 & 0,99 & 0,91 & 0,50 & 0,01 & 0,01 & 0,91 & 0,19 & 0,99 & 0,99 & 0,50 & 0,04 & 0,01 \\
\hline Combfd & 0,96 & 0,33 & 0,96 & 0,96 & 0,19 & 0,01 & 0,04 & 0,91 & 0,33 & 0,96 & 0,96 & 0,19 & 0,04 & 0,04 \\
\hline \multirow{2}{*}{ Zone } & T-1 & \multicolumn{3}{|c|}{$\mathbf{T}$} & \multicolumn{3}{|c|}{$\mathbf{T}+1$} & T-1 & \multicolumn{3}{|c|}{$\mathbf{T}$} & \multicolumn{3}{|c|}{$T+1$} \\
\hline & Mois 1 & Mois 1 & Mois 2 & Mois 3 & Mois 1 & Mois 2 & Mois 3 & Mois 1 & Mois 1 & Mois 2 & Mois 3 & Mois 1 & Mois 2 & Mois 3 \\
\hline AR4 & 0,41 & 0,41 & 0,41 & 0,41 & 0,74 & 0,74 & 0,74 & 0,26 & 0,26 & 0,26 & 0,26 & 0,74 & 0,74 & 0,74 \\
\hline AR_AIC & 0,41 & 0,41 & 0,41 & 0,41 & 0,59 & 0,59 & 0,59 & 0,41 & 0,41 & 0,41 & 0,41 & 0,59 & 0,59 & 0,59 \\
\hline GETS & 0,01 & 0,06 & 0,00 & 0,00 & 0,06 & 0,01 & 0,01 & 0,00 & 0,14 & 0,00 & 0,00 & 0,06 & 0,01 & 0,01 \\
\hline Combm & 0,14 & 0,41 & 0,14 & 0,06 & 0,14 & 0,41 & 0,59 & 0,26 & 0,26 & 0,26 & 0,06 & 0,14 & 0,26 & 0,41 \\
\hline Combmt & 0,14 & 0,41 & 0,14 & 0,14 & 0,14 & 0,41 & 0,59 & 0,26 & 0,26 & 0,26 & 0,14 & 0,14 & 0,41 & 0,41 \\
\hline Combme & 0,14 & 0,26 & 0,14 & 0,14 & 0,41 & 0,41 & 0,59 & 0,26 & 0,26 & 0,14 & 0,14 & 0,41 & 0,41 & 0,26 \\
\hline Combfd & 0,14 & 0,59 & 0,14 & 0,06 & 0,26 & 0,26 & 0,26 & 0,26 & 0,41 & 0,26 & 0,14 & 0,26 & 0,26 & 0,14 \\
\hline
\end{tabular}

explicatives; combm : moyenne des prédicteurs individuels, combmt : moyenne tronquée des prédicteurs individuels, combme : médiane des prédicteurs individuels, combfd : moyenne pondérée en fonction des erreurs passées des prédicteurs individuels. On reporte pour chacune des méthodes le RMSFE, le MAFE, les probabilités critiques du test de signe de Diebold et Mariano et les probabilités critiques du test de zone. 
Tableau 4b. Résultats des prévisions récursives et roulantes - Premiers résultats

\begin{tabular}{|c|c|c|c|c|c|c|c|c|c|c|c|c|c|c|}
\hline \multirow{3}{*}{ RMSFE } & \multicolumn{7}{|c|}{ RECURSIF } & \multicolumn{7}{|c|}{ ROULANT } \\
\hline & T-1 & \multicolumn{3}{|c|}{$\mathbf{T}$} & \multicolumn{3}{|c|}{$\mathrm{T}+1$} & T-1 & \multicolumn{3}{|c|}{$\mathbf{T}$} & \multicolumn{3}{|c|}{$T+1$} \\
\hline & Mois 1 & Mois 1 & Mois 2 & Mois 3 & Mois 1 & Mois 2 & Mois 3 & Mois 1 & Mois 1 & Mois 2 & Mois 3 & Mois 1 & Mois 2 & Mois 3 \\
\hline AR4 & $0,32 \%$ & $0,32 \%$ & $0,32 \%$ & $0,32 \%$ & $0,34 \%$ & $0,34 \%$ & $0,34 \%$ & $0,32 \%$ & $0,32 \%$ & $0,32 \%$ & $0,32 \%$ & $0,35 \%$ & $0,35 \%$ & $0,35 \%$ \\
\hline AR_AIC & $0,34 \%$ & $0,34 \%$ & $0,34 \%$ & $0,34 \%$ & $0,34 \%$ & $0,34 \%$ & $0,34 \%$ & $0,36 \%$ & $0,36 \%$ & $0,36 \%$ & $0,36 \%$ & $0,34 \%$ & $0,34 \%$ & $0,34 \%$ \\
\hline GETS & $0,25 \%$ & $0,27 \%$ & $0,23 \%$ & $0,23 \%$ & $0,33 \%$ & $0,39 \%$ & $0,35 \%$ & $0,26 \%$ & $0,26 \%$ & $0,23 \%$ & $0,22 \%$ & $0,32 \%$ & $0,37 \%$ & $0,34 \%$ \\
\hline Combm & $0,30 \%$ & $0,30 \%$ & $0,30 \%$ & $0,29 \%$ & $0,30 \%$ & $0,30 \%$ & $0,31 \%$ & $0,29 \%$ & $0,30 \%$ & $0,30 \%$ & $0,29 \%$ & $0,30 \%$ & $0,29 \%$ & $0,31 \%$ \\
\hline Combmt & $0,30 \%$ & $0,30 \%$ & $0,30 \%$ & $0,29 \%$ & $0,30 \%$ & $0,30 \%$ & $0,31 \%$ & $0,29 \%$ & $0,30 \%$ & $0,30 \%$ & $0,29 \%$ & $0,30 \%$ & $0,29 \%$ & $0,31 \%$ \\
\hline Combme & $0,30 \%$ & $0,30 \%$ & $0,31 \%$ & $0,30 \%$ & $0,30 \%$ & $0,30 \%$ & $0,32 \%$ & $0,30 \%$ & $0,29 \%$ & $0,30 \%$ & $0,30 \%$ & $0,30 \%$ & $0,29 \%$ & $0,31 \%$ \\
\hline Combfd & $0,29 \%$ & $0,29 \%$ & $0,29 \%$ & $0,29 \%$ & $0,31 \%$ & $0,31 \%$ & $0,31 \%$ & $0,29 \%$ & $0,28 \%$ & $0,29 \%$ & $0,29 \%$ & $0,30 \%$ & $0,30 \%$ & $0,31 \%$ \\
\hline \multirow{2}{*}{ MAFE } & T-1 & \multicolumn{3}{|c|}{$\mathbf{T}$} & \multicolumn{3}{|c|}{$\mathbf{T}+1$} & T-1 & \multicolumn{3}{|c|}{$\mathbf{T}$} & \multicolumn{3}{|c|}{$\mathbf{T}+1$} \\
\hline & Mois 1 & Mois 1 & Mois 2 & Mois 3 & Mois 1 & Mois 2 & Mois 3 & Mois 1 & Mois 1 & Mois 2 & Mois 3 & Mois 1 & Mois 2 & Mois 3 \\
\hline AR4 & $0,27 \%$ & $0,27 \%$ & $0,27 \%$ & $0,27 \%$ & $0,30 \%$ & $0,30 \%$ & $0,30 \%$ & $0,28 \%$ & $0,28 \%$ & $0,28 \%$ & $0,28 \%$ & $0,30 \%$ & $0,30 \%$ & $0,30 \%$ \\
\hline AR_AIC & $0,29 \%$ & $0,29 \%$ & $0,29 \%$ & $0,29 \%$ & $0,29 \%$ & $0,29 \%$ & $0,29 \%$ & $0,31 \%$ & $0,31 \%$ & $0,31 \%$ & $0,31 \%$ & $0,30 \%$ & $0,30 \%$ & $0,30 \%$ \\
\hline GETS & $0,19 \%$ & $0,21 \%$ & $0,19 \%$ & $0,18 \%$ & $0,27 \%$ & $0,31 \%$ & $0,28 \%$ & $0,20 \%$ & $0,21 \%$ & $0,19 \%$ & $0,17 \%$ & $0,26 \%$ & $0,31 \%$ & $0,27 \%$ \\
\hline Combm & $0,25 \%$ & $0,24 \%$ & $0,25 \%$ & $0,24 \%$ & $0,24 \%$ & $0,25 \%$ & $0,26 \%$ & $0,25 \%$ & $0,24 \%$ & $0,25 \%$ & $0,24 \%$ & $0,24 \%$ & $0,25 \%$ & $0,26 \%$ \\
\hline Combmt & $0,25 \%$ & $0,24 \%$ & $0,25 \%$ & $0,24 \%$ & $0,24 \%$ & $0,25 \%$ & $0,26 \%$ & $0,25 \%$ & $0,23 \%$ & $0,25 \%$ & $0,24 \%$ & $0,24 \%$ & $0,25 \%$ & $0,26 \%$ \\
\hline Combme & $0,25 \%$ & $0,23 \%$ & $0,25 \%$ & $0,25 \%$ & $0,25 \%$ & $0,24 \%$ & $0,26 \%$ & $0,25 \%$ & $0,23 \%$ & $0,25 \%$ & $0,25 \%$ & $0,24 \%$ & $0,24 \%$ & $0,26 \%$ \\
\hline Combfd & $0,24 \%$ & $0,23 \%$ & $0,24 \%$ & $0,24 \%$ & $0,25 \%$ & $0,26 \%$ & $0,27 \%$ & $0,24 \%$ & $0,22 \%$ & $0,25 \%$ & $0,24 \%$ & $0,24 \%$ & $0,25 \%$ & $0,26 \%$ \\
\hline \multirow{2}{*}{ Signe } & T-1 & \multicolumn{3}{|c|}{$\mathbf{T}$} & \multicolumn{3}{|c|}{$\mathbf{T}+1$} & T-1 & \multicolumn{3}{|c|}{$\mathbf{T}$} & \multicolumn{3}{|c|}{$\mathbf{T}+\mathbf{1}$} \\
\hline & Mois 1 & Mois 1 & Mois 2 & Mois 3 & Mois 1 & Mois 2 & Mois 3 & Mois 1 & Mois 1 & Mois 2 & Mois 3 & Mois 1 & Mois 2 & Mois 3 \\
\hline AR4 & 0,19 & 0,19 & 0,19 & 0,19 & 0,01 & 0,01 & 0,01 & 0,33 & 0,33 & 0,33 & 0,33 & 0,09 & 0,09 & 0,09 \\
\hline AR_AIC & 0,96 & 0,96 & 0,96 & 0,96 & 0,33 & 0,33 & 0,33 & 0,96 & 0,96 & 0,96 & 0,96 & 0,67 & 0,67 & 0,67 \\
\hline GETS & 0,09 & 0,00 & 0,01 & 0,00 & 0,09 & 0,50 & 0,04 & 0,19 & 0,00 & 0,01 & 0,00 & 0,04 & 0,09 & 0,09 \\
\hline Combm & 0,67 & 0,50 & 0,67 & 0,67 & 0,33 & 0,01 & 0,09 & 0,50 & 0,50 & 0,67 & 0,50 & 0,19 & 0,01 & 0,04 \\
\hline Combmt & 0,67 & 0,50 & 0,81 & 0,67 & 0,09 & 0,04 & 0,09 & 0,50 & 0,33 & 0,81 & 0,81 & 0,09 & 0,04 & 0,04 \\
\hline Combme & 0,67 & 0,67 & 0,91 & 0,50 & 0,09 & 0,04 & 0,19 & 0,50 & 0,50 & 0,81 & 0,81 & 0,09 & 0,09 & 0,19 \\
\hline Combfd & 0,50 & 0,33 & 0,67 & 0,67 & 0,19 & 0,04 & 0,09 & 0,19 & 0,19 & 0,67 & 0,67 & 0,19 & 0,04 & 0,09 \\
\hline \multirow{2}{*}{ Zone } & T-1 & \multicolumn{3}{|c|}{$\mathbf{T}$} & \multicolumn{3}{|c|}{$T+1$} & T-1 & \multicolumn{3}{|c|}{$\mathbf{T}$} & \multicolumn{3}{|c|}{$T+1$} \\
\hline & Mois 1 & Mois 1 & Mois 2 & Mois 3 & Mois 1 & Mois 2 & Mois 3 & Mois 1 & Mois 1 & Mois 2 & Mois 3 & Mois 1 & Mois 2 & Mois 3 \\
\hline AR4 & 0,14 & 0,14 & 0,14 & 0,14 & 0,14 & 0,14 & 0,14 & 0,06 & 0,06 & 0,06 & 0,06 & 0,14 & 0,14 & 0,14 \\
\hline AR_AIC & 0,02 & 0,02 & 0,02 & 0,02 & 0,06 & 0,06 & 0,06 & 0,41 & 0,41 & 0,41 & 0,41 & 0,06 & 0,06 & 0,06 \\
\hline GETS & 0,01 & 0,01 & 0,02 & 0,06 & 0,00 & 0,06 & 0,06 & 0,00 & 0,00 & 0,01 & 0,02 & 0,01 & 0,06 & 0,06 \\
\hline Combm & 0,02 & 0,02 & 0,02 & 0,06 & 0,01 & 0,02 & 0,06 & 0,06 & 0,01 & 0,06 & 0,06 & 0,01 & 0,01 & 0,02 \\
\hline Combmt & 0,02 & 0,02 & 0,02 & 0,02 & 0,01 & 0,02 & 0,06 & 0,06 & 0,01 & 0,06 & 0,02 & 0,01 & 0,02 & 0,02 \\
\hline Combme & 0,02 & 0,01 & 0,02 & 0,02 & 0,01 & 0,02 & 0,06 & 0,06 & 0,01 & 0,02 & 0,02 & 0,00 & 0,02 & 0,01 \\
\hline Combfd & 0,02 & 0,02 & 0,06 & 0,06 & 0,02 & 0,06 & 0,06 & 0,14 & 0,01 & 0,06 & 0,14 & 0,02 & 0,01 & 0,06 \\
\hline
\end{tabular}

Lecture : AR4 : processus autorégressif d'ordre fixé à 4, AR_AIC : processus autorégressif d'ordre variable basé sur le critère AIC, GETS : étalonnage univarié avec sélection automatique des variables
explicatives; combm : moyenne des prédicteurs individuels, combmt : movenne tronquée des prédicteurs individuels, combme : médiane des prédicteurs individuels, combfd : movenne pondérée en fonction des erreurs passées des prédicteurs individuels. On reporte pour chacune des méthodes le RMSFE, le MAFE, les probabilités critiques du test de signe de Diebold et Mariano et les probabilités critiques du test de zone. 


\section{Conclusion}

Dans cet article, nous avons examiné les performances de deux techniques alternatives pour prévoir le taux de croissance du PIB français sur de courts horizons : l'utilisation de modèles construits avec l'algorithme de sélection automatique GETS ou la combinaison d'un grand nombre d'étalonnages bivariés. Ces deux méthodes ont été adaptées de façon à prendre en compte l'évolution de l'ensemble d'information dans le trimestre.

Nous confirmons tout d'abord le gain à utiliser les enquêtes pour la prévision de court terme du PIB français, parmi lesquelles les enquêtes dans les services et dans le bâtiment. Nos résultats sont par ailleurs en faveur de l'utilisation de la méthode de sélection automatique plutôt que de la méthode de combinaison pour des étalonnages reposant uniquement sur des soldes d'enquêtes. Le gain est plus évident lorsque l'on évalue la capacité des modèles à reproduire les comptes définitifs que les premiers résultats, signe que les données d'enquête permettent peut-être d'anticiper les révisions du taux de croissance du PIB.

Plusieurs pistes sont envisageables pour prolonger ce travail. Il s'agira notamment d'examiner si l'analyse factorielle permet d'obtenir des prévisions de meilleure qualité que les deux approches retenues ici. On pourra également élargir le champ des enquêtes de conjoncture utilisées ici au commerce de détail et au commerce de gros, voire à l'enquête auprès des ménages. 


\section{Références}

Altavilla C. et Cicarelli M. (2007). "Information combination and forecast instability: Evidence from vintages of time-series data", ECB working paper No. 846.

Angelini E., Camba-Méndez G., Giannone D., Rünstler G. et Reichlin L. (2008). "Shortterm forecasts of euro area GDP growth", ECB working paper No. 949.

Bahroumi K., Brunhes-Lesage V., Darné O., Ferrara L., Pluyaud B. et Rouvreau B. (2008). "Monthly forecasting of French GDP : A revised version of the OPTIM model", Note d'Etudes et de Recherche de la Banque de France, No. 222, 2008.

Bates J.M. et Granger C.W.J. (1969). "The combination of forecasts", Operational Research Quarterly, 20, pp. 451-468.

Bouton F. et Erkel-Rousse H. (2002). "Conjonctures sectorielles et prévision à court terme de l'activité : L'apport de l'enquête de conjoncture dans les services", Economie et Statistique, 359-360, pp. 35-68.

Clark T.E. et McCracken M.W. (2004). "Improving forecast accuracy by combining recursive and rolling forecasts", Kansas City Federal Reserve. Working paper No. 04.10.

Croushore D. (2006). "Forecasting with real-time macroeconomic data", Handbook of Economic Forecasting, Elliott G., Granger C.W.J., Timmermann A. (eds). Amsterdam: Elsevier.

Darné O. et Brunhes-Lesage V. (2007). "L'indicateur synthétique mensuel d'activité (ISMA) : une révision", Note d'Etudes et de Recherche de la Banque de France, NER E\#171, 72 pages.

Diebold F.X. et Mariano R.S. (1995). "Comparing predictive accuracy”, Journal of Business and Economic Statistics, 13, pp. 253-263.

Doornik J.A. et Hansen H. (1994). "A practical test for univariate and multivariate normality", Discussion paper, Nuffield college.

Drechsel K. et Maurin L. (2008). "Flow of conjunctural information and forecast of euro area economic activity", ECB working paper No. 925.

Dubois E. et Michaux E. (2006). "Etalonnage à l'aide d'enquêtes de conjoncture : De nouveaux résultats", Economie et Prévision, 172, pp. 11-28.

Dubois E. et Michaux E. (2008). "GROCER 1.3 : an econometric toolbox for Scilab", Disponible sur http://dubois.ensae.net/grocer.html.

Erkel-Rousse H. et Minodier C. (2008). "Do Business Tendency Surveys in Industry and Services Help in Forecasting GDP Growth? A Real-Time Analysis on French Data", Association Française de Sciences Économiques, http://congres.afse.fr/docs/615069afse_erkelrousseminodier_soumissioncongres_20080423.pdf

Erkel-Rousse H. et Prioux G. (2002). "L'apport des enquêtes de conjoncture dans les différents secteurs d'activité à l'analyse conjoncturelle", Insee, Note de conjoncture de Juin, pp. 26-34.

Forni M., Hallin M., Lippi M. et Reichlin L. (2000). “The generalized dynamic factor model: Identification and estimation", The Review of Economics and Statistics, 82(4), pp. 540-552.

Forni M., Hallin M., Lippi M. et Reichlin L. (2005). "The generalized dynamic factor model: One-sided estimation and forecasting", Journal of the American Statistical Association, 471, pp. 830-52.

Giacomini R. et White H. (2006). "Tests of conditional predictive ability", Econometrica, 75, pp. 1545-78.

Godfrey L.G. (1978). "Testing for higher order serial correlation in regression equations when the regressors include lagged dependent variables", Econometrica, 46, pp. 1303-1313. 
Hendry D.F. et Krolzig H-M. (1999). "Improving on data mining reconsidered by K.D", Hoover and S.J. Perez. Econometric Journal, 2, pp. 41-58.

Hendry D.F. et Krolzig H-M. (2001). "Computer automation of general-to-specific model selection procedures", Journal of Economic Dynamic and Control, 25(6-7), pp. 831-866.

Hendry D.F. et Krolzig H-M. (2005). "The properties of automatic GETS modelling", The Economic Journal, 115 (March), C32-C61.

Heyer E. et Péléraux H. (2004). "Un indicateur de croissance infra-annuelle pour l'économie française", Revue de l'OFCE, 88, pp. 203-218.

Heyer E., Blot C., Cochard M., Péléraux H. et Plane M. (2008). "France : Entrée dans le tunnel ? Scénarios 2008-09 pour l'économie française", Revue de l'OFCE, 107, pp. 175-248.

Hoover K.D. et Perez S.J. (1999). "Data mining reconsidered: a general to specification approach to specification search", Econometric Journal, 2, pp. 167-191.

Kitchen J. et Monaco R. (2003). "Real-time forecasting in practice", Business Economics, 38(4), pp. 10-19.

Marcellino M. (2008). “A linear benchmark for forecasting GDP growth and inflation”, Journal of Forecasting, 27, pp. 305-340.

Marcellino M., Stock J.H. et Watson M.W. (2006). "A comparison of direct and iterated multistep AR methods for forecasting macroeconomic time series", Journal of Econometrics, 135(1-2), pp. 499-526.

Nicholls D.F. et Pagan A.R. (1983). "Heteroscedasticity in models with lagged dependent variables", Econometrica, 51, pp. 1233-1242.

Reid D.J. (1968). "Combining three estimates of gross domestic output", Economica, 35, pp. 431-444.

Sédillot F. et Pain N. (2005). "Modèles d'indicateurs de la croissance du PIB réel dans les principales économies de l'OCDE”, Revue Economique de l'OCDE, 40, pp. 185-246.

Stock J.H. et Watson M.W. (1999). "Forecasting inflation", Journal of Monetary Economics, 44, pp. 293-335.

Stock J.H. et Watson M.W. (2002). "Macroeconomic forecasting using diffusion indexes", Journal of Business and Economic Statistics, 20, pp. 147-162.

Stock J.H. et Watson M.W. (2004). "Combination forecasts of output growth in a sevencountry data set", Journal of Forecasting, 23(6), pp. 405-430. 


\section{Annexe 1}

\section{Les enquêtes de conjoncture de l'Insee}

\section{1) Enquêtes dans l'industrie}

* enquête mensuelle de conjoncture dans l'industrie (initiée en 1962)

* enquête trimestrielle de conjoncture dans l'industrie

- enquête trimestrielle sur les investissements dans l'industrie (initiée en 1963)

- enquête de trésorerie dans l'industrie (semestrielle, produite les mois de juin et décembre, réalisée pour la première fois en 1962)

2) Enquêtes dans les services

* enquête mensuelle de conjoncture dans les services (trimestrielle jusqu'au deuxième trimestre de 2000, initiée en 1988)

\section{3) Enquêtes dans la construction}

* enquête mensuelle de conjoncture dans le bâtiment (trimestrielle jusqu'au troisième trimestre de 1993, initiée en 1975)

- enquête trimestrielle dans la construction immobilière (initiée en 1969)

- enquête trimestrielle dans les travaux publics (initiée en 1974)

- enquête trimestrielle dans l'artisanat du bâtiment (initiée en 1990)

\section{4) Enquête dans le commerce}

- enquête bimestrielle de conjoncture dans le commerce de gros (réalisée les mois impairs depuis 1972)

- enquête mensuelle de conjoncture dans le commerce de détail et le commerce automobile (de 1964 à 1973, l'enquête avait lieu 5 fois par an ; en 1973, elle devient bimestrielle ; depuis la fin 1990, l'enquête est mensuelle)

5) Enquête auprès des ménages

- enquête mensuelle de conjoncture auprès des ménages (initiée en 1958 à une fréquence quadrimestrielle, une version mensuelle existe depuis janvier 1987)

6) International (retraitement des enquêtes d'autres pays)

- enquêtes européennes de conjoncture mensuelle

- enquêtes européennes de conjoncture trimestrielle

Les enquêtes utilisées dans cette étude sont précédées d'un astérisque. 


\section{Annexe 2}

\section{Contribution des trois enquêtes à l'estimation du taux de croissance trimestriel du PIB}

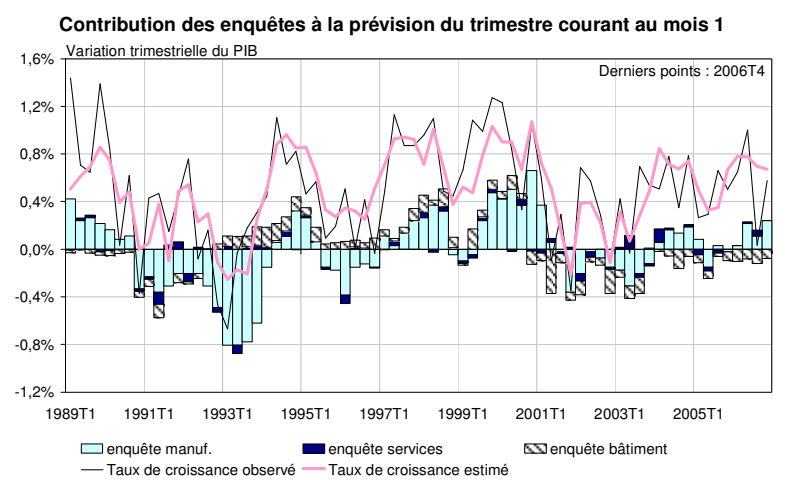

Contribution des enquêtes à la prévision du trimestre courant au mois 2

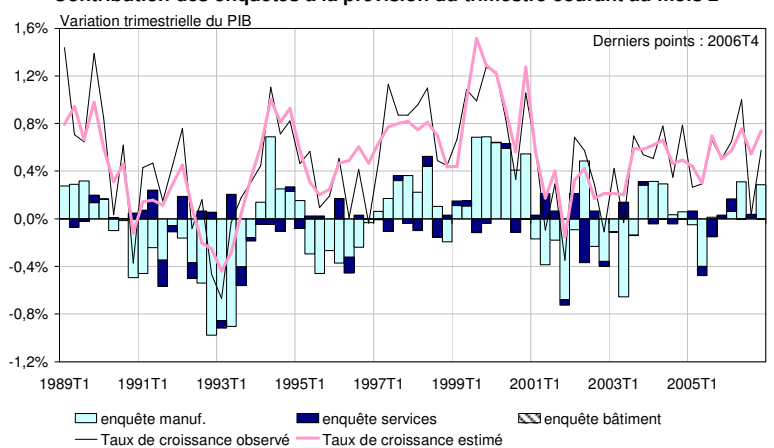

Contribution des enquêtes à la prévision du trimestre courant au mois 3 $1,6 \%$ Variation trimestrielle du PIB

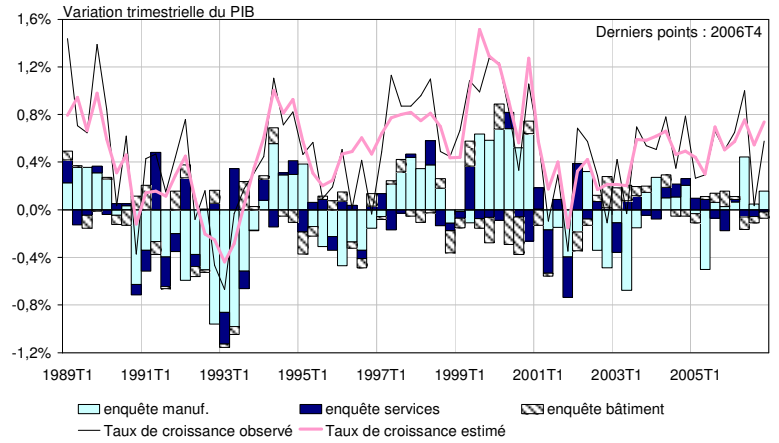

Contribution des enquêtes à la prévision du trimestre suivant au mois 1

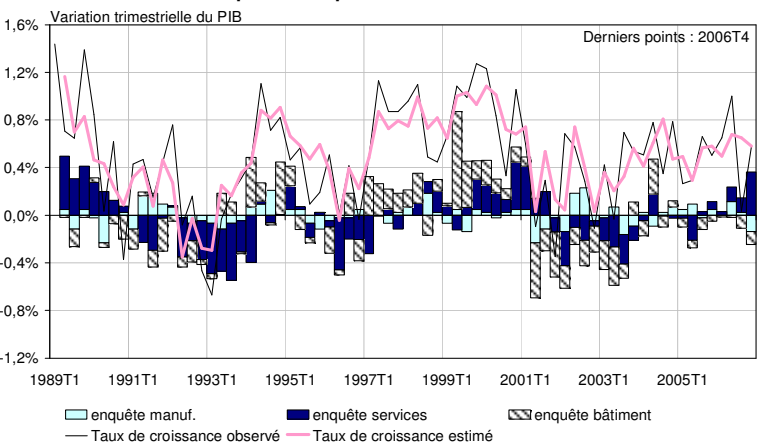

Contribution des enquêtes à la prévision du trimestre suivant au mois 2

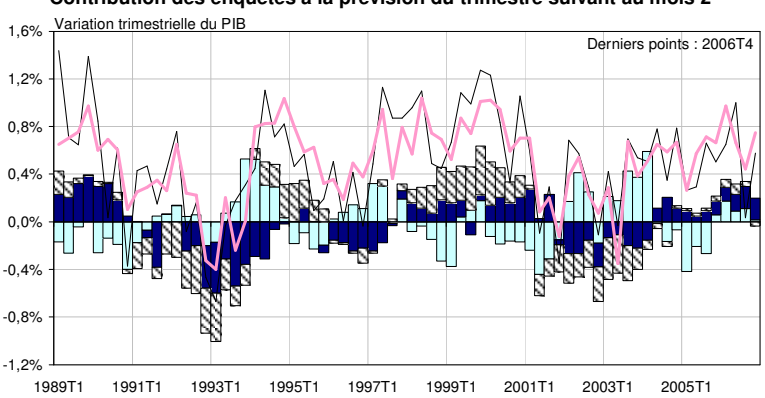
—enquête manuf. $\quad$ enquête services

Contribution des enquêtes à la prévision du trimestre suivant au mois 3

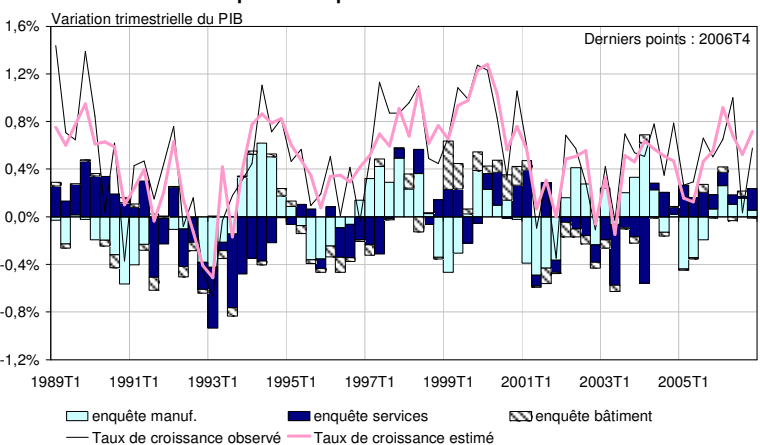

Lecture : la contribution d'un secteur à la variation du taux de croissance estimé en t autour de sa moyenne est obtenue en sommant les soldes centrés du secteur observés en t multipliés par leur poids estimé dans l'équation (nous utilisons ici l'équation centrée afin de ne pas avoir à représenter la contribution de la constante).

Tableau a2. Part des contributions des trois secteurs

\begin{tabular}{|c|c|c|c|c|}
\hline Trim. Prévu & Mois & Industrie & Services & Bâtiment \\
\hline $\mathrm{T}-1$ & 1 & $45 \%$ & $21 \%$ & $33 \%$ \\
\hline \multirow{3}{*}{$\mathrm{T}$} & 1 & $64 \%$ & $9 \%$ & $27 \%$ \\
\cline { 2 - 5 } & 2 & $77 \%$ & $23 \%$ & $0 \%$ \\
\cline { 2 - 5 } & 3 & $56 \%$ & $24 \%$ & $20 \%$ \\
\hline \multirow{3}{*}{$\mathrm{T}+1$} & 1 & $20 \%$ & $41 \%$ & $39 \%$ \\
\cline { 2 - 5 } & 2 & $34 \%$ & $34 \%$ & $31 \%$ \\
\cline { 2 - 5 } & 3 & $46 \%$ & $42 \%$ & $12 \%$ \\
\hline \multirow{3}{*}{ Moyenne } & $\mathrm{T}-1$ et T & $61 \%$ & $19 \%$ & $20 \%$ \\
\cline { 2 - 5 } & $\mathrm{T}$ & $66 \%$ & $19 \%$ & $28 \%$ \\
\cline { 2 - 5 } & $\mathrm{T}+1$ & $33 \%$ & $39 \%$ & $28 \%$ \\
\hline
\end{tabular}

Lecture : Ce tableau présente la moyenne sur la période 1988T2 à 2006T4 des parts des contributions en valeur absolue de chaque secteur à la somme de ces contributions prises en valeur absolue. 


\section{Annexe 3}

Résultats en-échantillon (1991T1 à 2006T4)

\begin{tabular}{|c|c|c|c|c|c|c|c|c|c|c|c|c|c|c|}
\hline \multirow{3}{*}{ RMSE } & \multicolumn{7}{|c|}{ COMPTES DEFINITIFS } & \multicolumn{7}{|c|}{ PREMIERS RESULTATS } \\
\hline & T-1 & \multicolumn{3}{|c|}{$\mathbf{T}$} & \multicolumn{3}{|c|}{$\mathbf{T}+1$} & T-1 & \multicolumn{3}{|c|}{$\mathbf{T}$} & \multicolumn{3}{|c|}{$T+1$} \\
\hline & Mois 1 & Mois 1 & Mois 2 & Mois 3 & Mois 1 & Mois 2 & Mois 3 & Mois 1 & Mois 1 & Mois 2 & Mois 3 & Mois 1 & Mois 2 & Mois 3 \\
\hline AR4 & $0,36 \%$ & $0,36 \%$ & $0,36 \%$ & $0,36 \%$ & $0,38 \%$ & $0,38 \%$ & $0,38 \%$ & $0,35 \%$ & $0,35 \%$ & $0,35 \%$ & $0,35 \%$ & $0,37 \%$ & $0,37 \%$ & $0,37 \%$ \\
\hline GETS & $0,21 \%$ & $0,28 \%$ & $0,22 \%$ & $0,22 \%$ & $0,27 \%$ & $0,26 \%$ & $0,22 \%$ & $0,31 \%$ & $0,34 \%$ & $0,31 \%$ & $0,35 \%$ & $0,32 \%$ & $0,35 \%$ & $0,34 \%$ \\
\hline Combm & $0,31 \%$ & $0,32 \%$ & $0,31 \%$ & $0,31 \%$ & $0,34 \%$ & $0,32 \%$ & $0,33 \%$ & $0,33 \%$ & $0,34 \%$ & $0,33 \%$ & $0,33 \%$ & $0,36 \%$ & $0,34 \%$ & $0,34 \%$ \\
\hline Combmt & $0,31 \%$ & $0,31 \%$ & $0,31 \%$ & $0,31 \%$ & $0,35 \%$ & $0,32 \%$ & $0,33 \%$ & $0,33 \%$ & $0,34 \%$ & $0,33 \%$ & $0,33 \%$ & $0,36 \%$ & $0,34 \%$ & $0,33 \%$ \\
\hline Combme & $0,31 \%$ & $0,32 \%$ & $0,32 \%$ & $0,31 \%$ & $0,35 \%$ & $0,33 \%$ & $0,33 \%$ & $0,33 \%$ & $0,34 \%$ & $0,33 \%$ & $0,33 \%$ & $0,36 \%$ & $0,34 \%$ & $0,35 \%$ \\
\hline Combfd & $0,31 \%$ & $0,32 \%$ & $0,31 \%$ & $0,31 \%$ & $0,35 \%$ & $0,32 \%$ & $0,33 \%$ & $0,33 \%$ & $0,34 \%$ & $0,33 \%$ & $0,33 \%$ & $0,36 \%$ & $0,34 \%$ & $0,34 \%$ \\
\hline \multirow{2}{*}{ MAE } & T-1 & \multicolumn{3}{|c|}{$\mathbf{T}$} & \multicolumn{3}{|c|}{$\mathrm{T}+1$} & T-1 & \multicolumn{3}{|c|}{$\mathbf{T}$} & \multicolumn{3}{|c|}{$\mathbf{T}+\mathbf{1}$} \\
\hline & Mois 1 & Mois 1 & Mois 2 & Mois 3 & Mois 1 & Mois 2 & Mois 3 & Mois 1 & Mois 1 & Mois 2 & Mois 3 & Mois 1 & Mois 2 & Mois 3 \\
\hline AR4 & $0,29 \%$ & $0,29 \%$ & $0,29 \%$ & $0,29 \%$ & $0,31 \%$ & $0,31 \%$ & $0,31 \%$ & $0,28 \%$ & $0,28 \%$ & $0,28 \%$ & $0,28 \%$ & $0,30 \%$ & $0,30 \%$ & $0,30 \%$ \\
\hline GETS & $0,16 \%$ & $0,24 \%$ & $0,16 \%$ & $0,17 \%$ & $0,21 \%$ & $0,21 \%$ & $0,16 \%$ & $0,24 \%$ & $0,27 \%$ & $0,24 \%$ & $0,25 \%$ & $0,25 \%$ & $0,28 \%$ & $0,27 \%$ \\
\hline Combm & $0,25 \%$ & $0,26 \%$ & $0,25 \%$ & $0,25 \%$ & $0,27 \%$ & $0,26 \%$ & $0,26 \%$ & $0,26 \%$ & $0,26 \%$ & $0,26 \%$ & $0,26 \%$ & $0,28 \%$ & $0,26 \%$ & $0,27 \%$ \\
\hline Combmt & $0,25 \%$ & $0,25 \%$ & $0,25 \%$ & $0,25 \%$ & $0,28 \%$ & $0,26 \%$ & $0,27 \%$ & $0,26 \%$ & $0,26 \%$ & $0,26 \%$ & $0,26 \%$ & $0,28 \%$ & $0,26 \%$ & $0,26 \%$ \\
\hline Combme & $0,26 \%$ & $0,26 \%$ & $0,26 \%$ & $0,25 \%$ & $0,28 \%$ & $0,26 \%$ & $0,26 \%$ & $0,26 \%$ & $0,26 \%$ & $0,26 \%$ & $0,26 \%$ & $0,29 \%$ & $0,27 \%$ & $0,27 \%$ \\
\hline Combfd & $0,25 \%$ & $0,26 \%$ & $0,25 \%$ & $0,25 \%$ & $0,28 \%$ & $0,26 \%$ & $0,26 \%$ & $0,26 \%$ & $0,26 \%$ & $0,26 \%$ & $0,26 \%$ & $0,28 \%$ & $0,26 \%$ & $0,27 \%$ \\
\hline \multirow{2}{*}{ Signe } & T-1 & \multicolumn{3}{|c|}{$\mathbf{T}$} & \multicolumn{3}{|c|}{$T+1$} & T-1 & \multicolumn{3}{|c|}{$\mathbf{T}$} & \multicolumn{3}{|c|}{$T+1$} \\
\hline & Mois 1 & Mois 1 & Mois 2 & Mois 3 & Mois 1 & Mois 2 & Mois 3 & Mois 1 & Mois 1 & Mois 2 & Mois 3 & Mois 1 & Mois 2 & Mois 3 \\
\hline AR4 & 0,98 & 0,98 & 0,98 & 0,98 & 0,26 & 0,26 & 0,26 & 0,98 & 0,98 & 0,98 & 0,98 & 0,26 & 0,26 & 0,26 \\
\hline GETS & 0,00 & 0,00 & 0,00 & 0,00 & 0,00 & 0,01 & 0,00 & 0,00 & 0,00 & 0,00 & 0,00 & 0,00 & 0,01 & 0,00 \\
\hline Combm & 0,26 & 0,05 & 0,55 & 0,45 & 0,08 & 0,08 & 0,13 & 0,26 & 0,05 & 0,55 & 0,45 & 0,08 & 0,08 & 0,13 \\
\hline Combmt & 0,45 & 0,45 & 0,55 & 0,45 & 0,08 & 0,13 & 0,05 & 0,45 & 0,08 & 0,55 & 0,45 & 0,08 & 0,13 & 0,55 \\
\hline Combme & 0,55 & 0,05 & 0,65 & 0,65 & 0,13 & 0,05 & 0,05 & 0,55 & 0,13 & 0,65 & 0,65 & 0,13 & 0,05 & 0,08 \\
\hline Combfd & 0,26 & 0,03 & 0,55 & 0,45 & 0,08 & 0,08 & 0,13 & 0,26 & 0,03 & 0,55 & 0,45 & 0,08 & 0,08 & 0,13 \\
\hline \multirow{2}{*}{ Zone } & T-1 & \multicolumn{3}{|c|}{$\mathbf{T}$} & \multicolumn{3}{|c|}{$\mathbf{T}+1$} & T-1 & \multicolumn{3}{|c|}{$\mathbf{T}$} & \multicolumn{3}{|c|}{$\mathbf{T}+1$} \\
\hline & Mois 1 & Mois 1 & Mois 2 & Mois 3 & Mois 1 & Mois 2 & Mois 3 & Mois 1 & Mois 1 & Mois 2 & Mois 3 & Mois 1 & Mois 2 & Mois 3 \\
\hline AR4 & 0,07 & 0,07 & 0,07 & 0,07 & 0,11 & 0,11 & 0,11 & 0,07 & 0,07 & 0,07 & 0,07 & 0,11 & 0,11 & 0,11 \\
\hline GETS & 0,00 & 0,00 & 0,00 & 0,00 & 0,00 & 0,00 & 0,00 & 0,00 & 0,00 & 0,00 & 0,00 & 0,00 & 0,00 & 0,00 \\
\hline Combm & 0,00 & 0,00 & 0,00 & 0,00 & 0,00 & 0,00 & 0,00 & 0,00 & 0,00 & 0,00 & 0,00 & 0,00 & 0,00 & 0,00 \\
\hline Combmt & 0,00 & 0,00 & 0,00 & 0,00 & 0,00 & 0,00 & 0,00 & 0,00 & 0,00 & 0,00 & 0,00 & 0,00 & 0,00 & 0,00 \\
\hline Combme & 0,00 & 0,00 & 0,00 & 0,00 & 0,00 & 0,00 & 0,00 & 0,00 & 0,00 & 0,00 & 0,00 & 0,00 & 0,00 & 0,00 \\
\hline Combfd & 0,00 & 0,00 & 0,00 & 0,00 & 0,00 & 0,00 & 0,00 & 0,00 & 0,00 & 0,00 & 0,00 & 0,00 & 0,00 & 0,00 \\
\hline
\end{tabular}




\section{Annexe 4}

\section{Prévision du trimestre suivant $\mathbf{T}+1$}

\section{Prévision du trimestre suivant au mois $\mathbf{3}$ - méthode de combinaison}

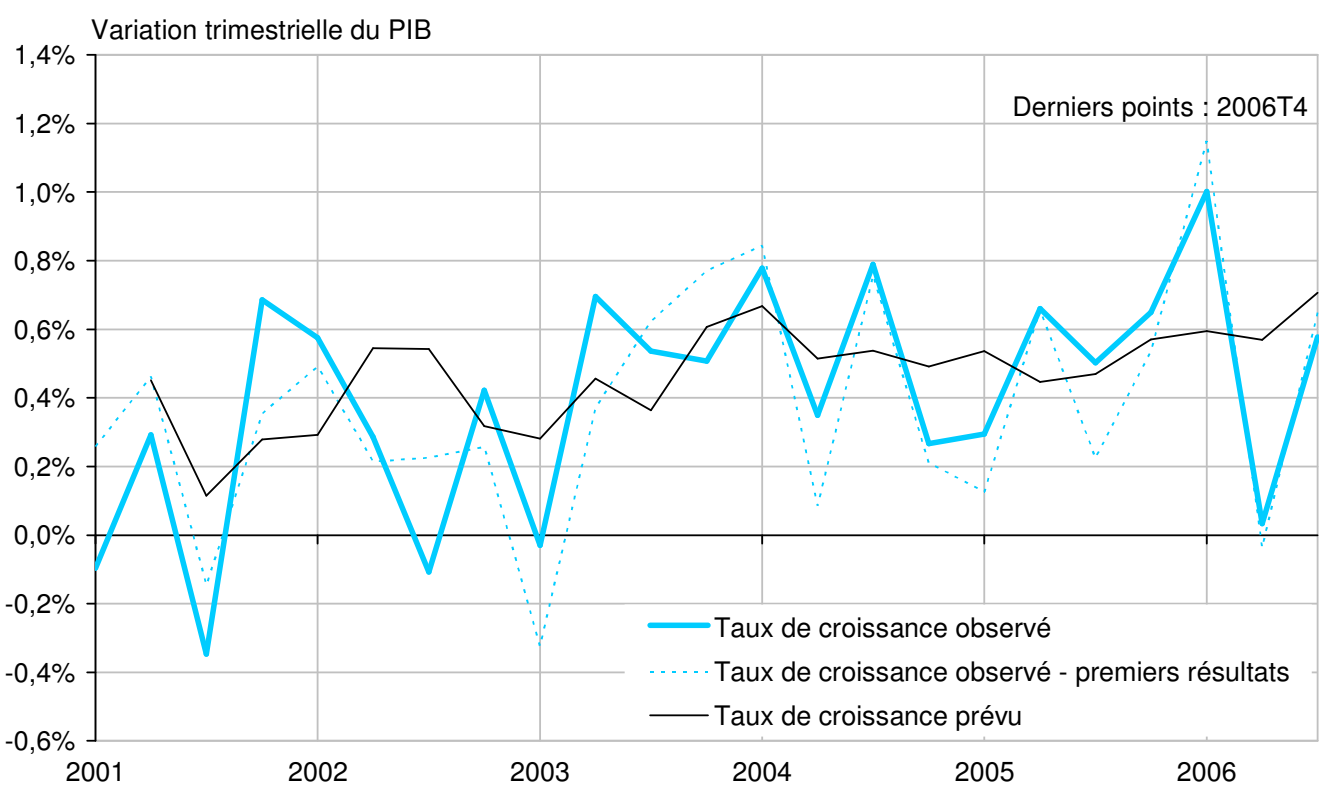

Prévision du trimestre suivant au mois 3 - méthode GETS

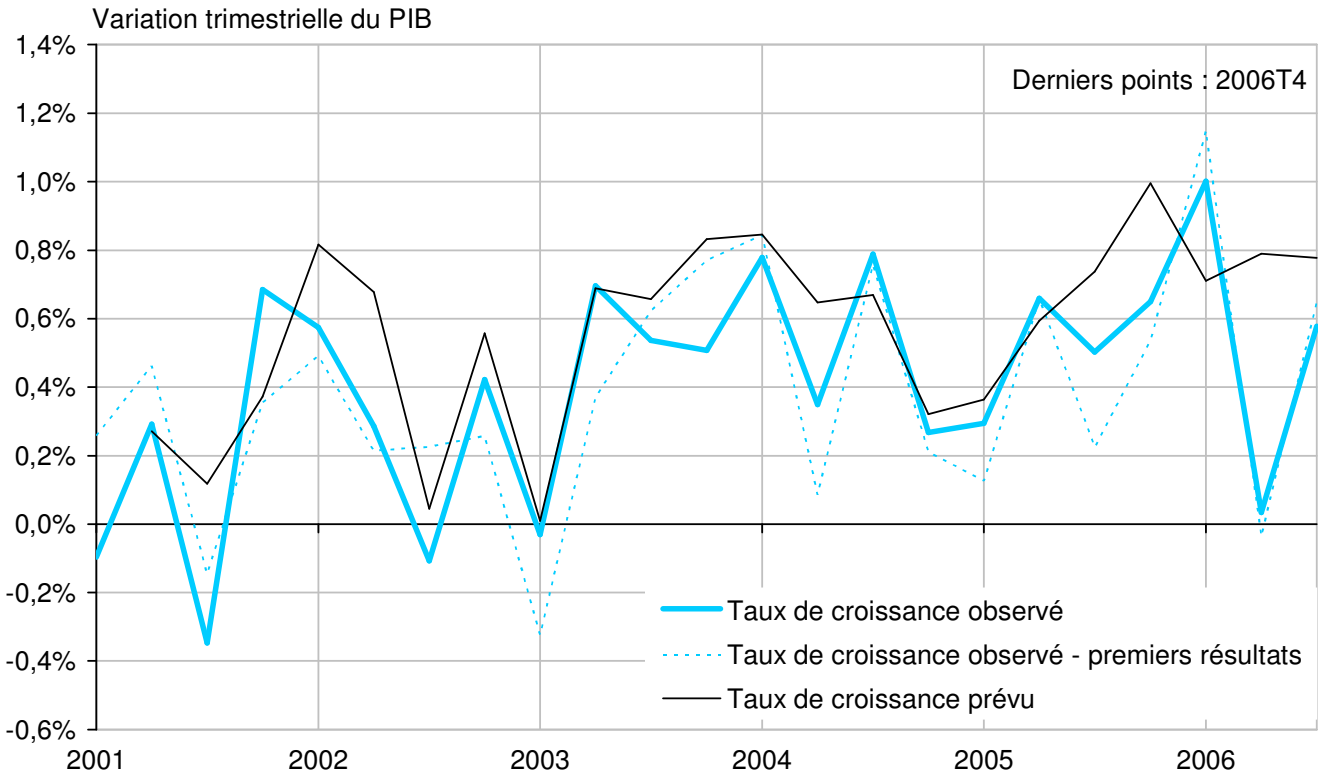

